livraisons

d'Histoire

de l'Architecture

\section{Livraisons de l'histoire de l'architecture}

13 | 2007

Architectures des établissements d'enseignement supérieur

\title{
Jussieu, l'inachevée. Cinquante ans de projets pour la « faculté des sciences de Paris centre »
}

The incomplete Jussieu : fifty years of projects for the Faculty of Science in Paris", by Christian Hottin

Der unvollendete Bau von Jussieu : fünfzigjähriges Projekt für die Fakultät der Wissenschaften Paris-Centre

\section{Christian Hottin}

\section{OpenEdition Journals}

Édition électronique

URL : http://journals.openedition.org/lha/402

DOI : $10.4000 /$ lha. 402

ISSN : 1960-5994

Éditeur

Association Livraisons d'histoire de l'architecture - LHA

Édition imprimée

Date de publication : 10 juin 2007

Pagination : $23-50$

ISSN : 1627-4970

Référence électronique

Christian Hottin, « Jussieu, l'inachevée. Cinquante ans de projets pour la « faculté des sciences de Paris centre » ", Livraisons de l'histoire de l'architecture [En ligne], 13 | 2007, mis en ligne le 10 juin 2009, consulté le 01 mai 2019. URL : http://journals.openedition.org//ha/402 ; DOI : 10.4000//ha.402

Ce document a été généré automatiquement le 1 mai 2019.

Tous droits réservés à l'Association LHA 


\title{
Jussieu, l'inachevée. Cinquante ans de projets pour la « faculté des sciences de Paris centre»
}

\author{
The incomplete Jussieu : fifty years of projects for the Faculty of Science in \\ Paris", by Christian Hottin \\ Der unvollendete Bau von Jussieu : fünfzigjähriges Projekt für die Fakultät der \\ Wissenschaften Paris-Centre
}

\section{Christian Hottin}

«...Mais il faut agir vite. Car une faculté des sciences doit se construire en trois ans pour trente ans et non en trente ans pour trois ans ", Marc Zamansky, doyen de la Faculté des sciences de Paris, novembre $1961^{1}$

«Les sept merveilles de la France » : telle est la formule choisie par Xavier Laurent ${ }^{2}$ pour désigner les monuments du patrimoine national qui furent l'objet de toutes les attentions de la république gaullienne. La liste des sept merveilles de la France des Trente Glorieuses reste à dresser. On y trouverait sans doute, à côté de La Défense et de la Maison de la radio, le paquebot France et l'usine marémotrice de la Rance, le Concorde ou le pont de Tancarville, en prenant soin d'omettre les abattoirs de La Villette. On ne saurait oublier dans cette liste la Faculté des sciences de Paris - centre, puisque tel fut son nom lorsque, débarrassée de ses origines mercantiles (la «faculté de la Halle aux vins »), elle n'était pas encore réduite par métonymie au nom de la station de métro la plus proche ( Jussieu »). La variation toponymique est comme l'écho assourdi de l'enlisement précoce d'un projet qui, après une gestation d'un siècle ${ }^{3}$, connaît en 1962 une naissance glorieuse suivie d'une enfance fastueuse ${ }^{4}$, puis, dès 1973 , vingt années d'abandon, d'hésitations et de promesses non tenues, jusqu'à ce que, en 1996, le scandale de l'amiante ne le tire de l'oubli pour poser de manière brutale la question de la destruction ou de l'achèvement des locaux. Dix ans passent encore, et l'année 2007 voit l'inauguration à Tolbiac des nouveaux bâtiments 
de l'Université Paris VII - Denis Diderot, tandis que se poursuivent à Jussieu le désamiantage des locaux de Paris VI - Pierre et Marie Curie, la rénovation des barres existantes et le parachèvement architectural du site. Happy end pour Jussieu?

2 À l'intérieur de cette brève séquence temporelle - à peine cinquante ans - se dessine le schéma optimiste d'une suite de trois phases : grandeur, décadence et résurrection d'une grande idée. En faire le récit n'épuise pas le sujet et tendrait même plutôt à le réduire, à l'isoler à outrance. La singularité de l'architecture y invite du reste: si Jussieu a été, depuis trente ans, un cauchemar pour les universitaires et nombre de défenseurs du patrimoine, il est au contraire apparu, pour les architectes comme pour les historiens de l'architecture contemporaine, un objet de rêve. Plusieurs grands noms de la création contemporaine, aux premiers rangs desquels Jean Nouvel et Rem Koolhaas, ont cherché à affronter la cohérence absolue du parti défini par Édouard Albert, cette trame qui constitue un défi pour tout continuateur éventuel. Pourtant, comme on l'a suggéré ailleurs ${ }^{5}$, nous pensons que l'exemple de Jussieu doit être mis en relation avec d'autres grands projets universitaires morts-nés, corsetés ou amputés qui forment l'armature de l'histoire matérielle chaotique de l'université française depuis deux siècles : à travers eux se lit l'inconstance des politiques publiques successives, les frustrations de l'institution, la mise en place d'une identité problématique et, bien entendu, la difficile réalisation de ses ambitions scientifiques.

\section{Du lancement à l'enlisement (1962-1972)}

3 Interpréter les politiques publiques et les programmes architecturaux élaborés depuis plus de trente ans pour " parachever » - selon l'expression consacrée pour désigner ce problème ${ }^{6}$ - le " campus » - ce qu'il n'est pas au sens propre du terme - de Jussieu, nécessite au préalable de comprendre la logique formelle et fonctionnelle du projet initial et son articulation avec les vues administratives et scientifiques de l'institution universitaire dans les années soixante. En effet, "Jussieu l'inachevée», ce n'est pas seulement la réalisation incomplète du projet architectural mis au point par Édouard Albert. De fait, l'abandon du dessein institutionnel conçu par l'administration de l'enseignement supérieur et son remplacement, après 1970, par l'organisation qui régit aujourd'hui encore le système universitaire francilien sont deux décisions qui créent une distorsion durable et profonde entre la partie construite de l'œuvre architecturale et les établissements qui l'occupent (par voie de conséquence leurs agents en sont également affectés). Ainsi, derrière l'inachèvement (ou plus exactement les inachèvements) apparaît l'inadéquation fondamentale entre le contenant et le contenu du lieu, entre sa forme et ses fonctions, et se devinent les tensions et les rapports de force entre institutions rivales qui ont pour objet et enjeu cet espace inapproprié.

\section{Quatre essais non transformés}

4 La radicalité du projet établi par Édouard Albert en octobre 1962 apparaît mieux encore quand on le compare aux solutions imaginées au cours des vingt années précédentes pour l'implantation sur le site de la Halle aux vins de la Faculté des sciences. Dès 1942, le doyen Paul Montel demande à Roger Séassal, architecte de la Faculté, un projet pour le site : Il ne peut être exécuté en raison de la guerre (ill. 1). 


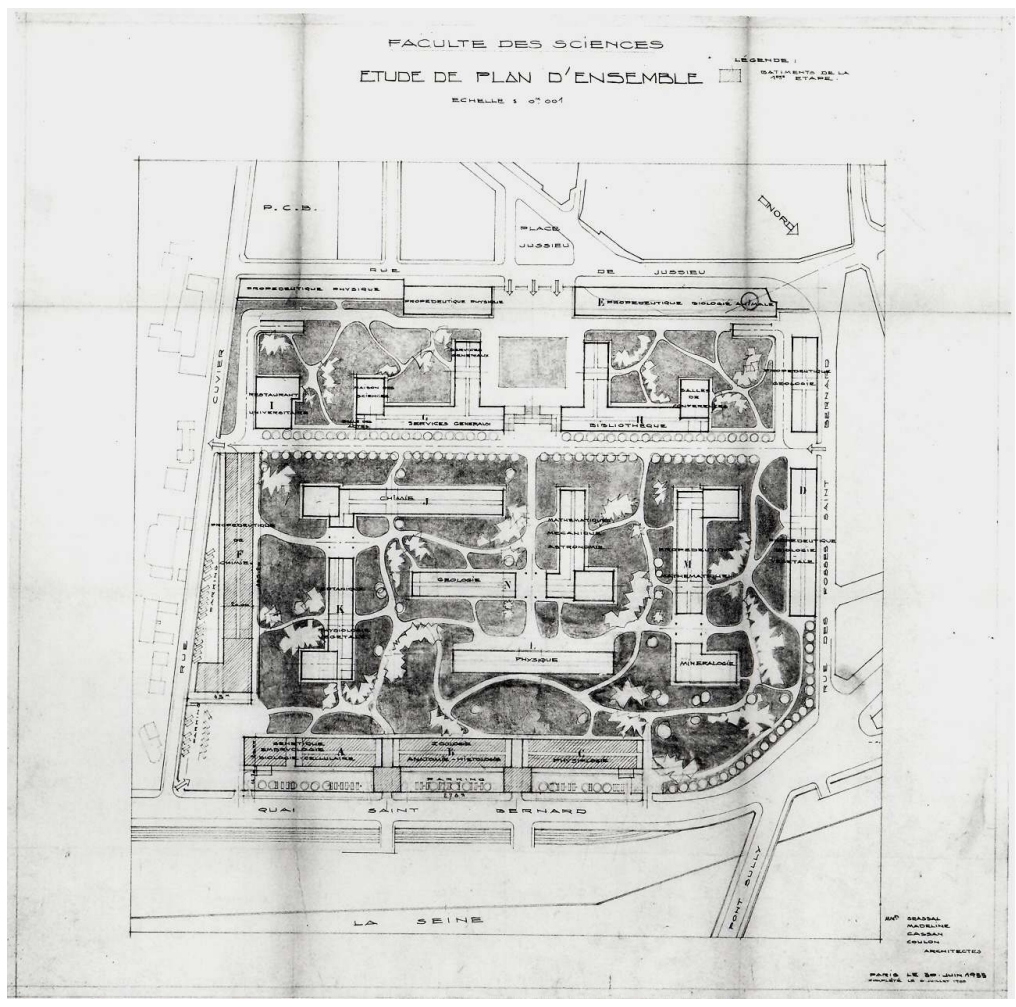

Projet pour la Faculté des sciences de Paris sur le site de la Halle aux vins, plan masse, 30 juin 1955. Arch. nat. (C.H.A.N.), F 216673

$\mathrm{Cl}$. Archives nationales.

En 1955, la Faculté a enfin pris pied sur le site : une modeste aile de deux étages s'élève sur pilotis au-dessus des chais de la maison Dubonnet ${ }^{7}$. Deux autres bâtiments, de plus grandes dimensions et formant une équerre, sont projetés le long de la rue Cuvier et sur le quai Saint-Bernard ${ }^{8}$. Là encore, la construction sur pilotis doit permettre aux négociants de poursuivre l'exploitation de leurs concessions une fois les locaux achevés. Urbain Cassan, Louis Madeline et René Coulon sont les trois architectes que le ministre Jean-Marie Berthoin a désignés pour œuvrer avec Séassal. Le 30 juin 1955 ils établissement un premier plan d'ensemble, modifié le 30 avril $1956^{10}$. Dans un cas comme dans l'autre, les architectes adoptent une configuration qui ne sera plus remise en cause : l'entrée principale de la faculté est située au sud, face à la place Jussieu. Pour le reste, et faute de connaître les élévations des bâtiments projetés, il semble qu'ils s'éloignent moins qu'on ne pourrait le penser du projet de Séassal. Sur l'un comme l'autre plan, le site est divisé en deux parties d'inégales superficies. Le tiers méridional du terrain occupe une position plus élevée que la partie tournée vers le fleuve, la déclivité naturelle du site est soulignée par des escaliers monumentaux qui ferment la cour d'honneur. Le dernier projet élaboré par cette équipe, celui de 1961, marque plus nettement encore leur attachement aux grandes masses indépendantes, puisqu'ils envisagent quatre barres perpendiculaires au bâtiment $\mathrm{ABC}$, longues de cent soixante-quatre mètres et hautes de quelques soixante-dix mètres ${ }^{11}$.

6 Selon Bernard Marrey, il semble bien que la visite du chantier par André Malraux en 1962, alors que Madeline vient de mourir, ait été l'occasion pour le ministre de se saisir du 
dossier et d'inclure Édouard Albert dans l'équipe, non sans l'accord du nouveau doyen Marc Zamansky.

\section{Une triple singularité}

7 Venant après ces années d'hésitations et de réalisations partielles, le projet mis au point entre octobre 1962 et mars 1963 se distingue par la cohérence de son parti, jointe à son inscription dans un programme institutionnel de grande ampleur. Enfin, l'implication personnelle d'André Malraux lui confère une valeur exceptionnelle et inédite (ill. 2).

\section{2 : Édouard Albert, Urbain Cassan, René Coulon et Roger Séassal}

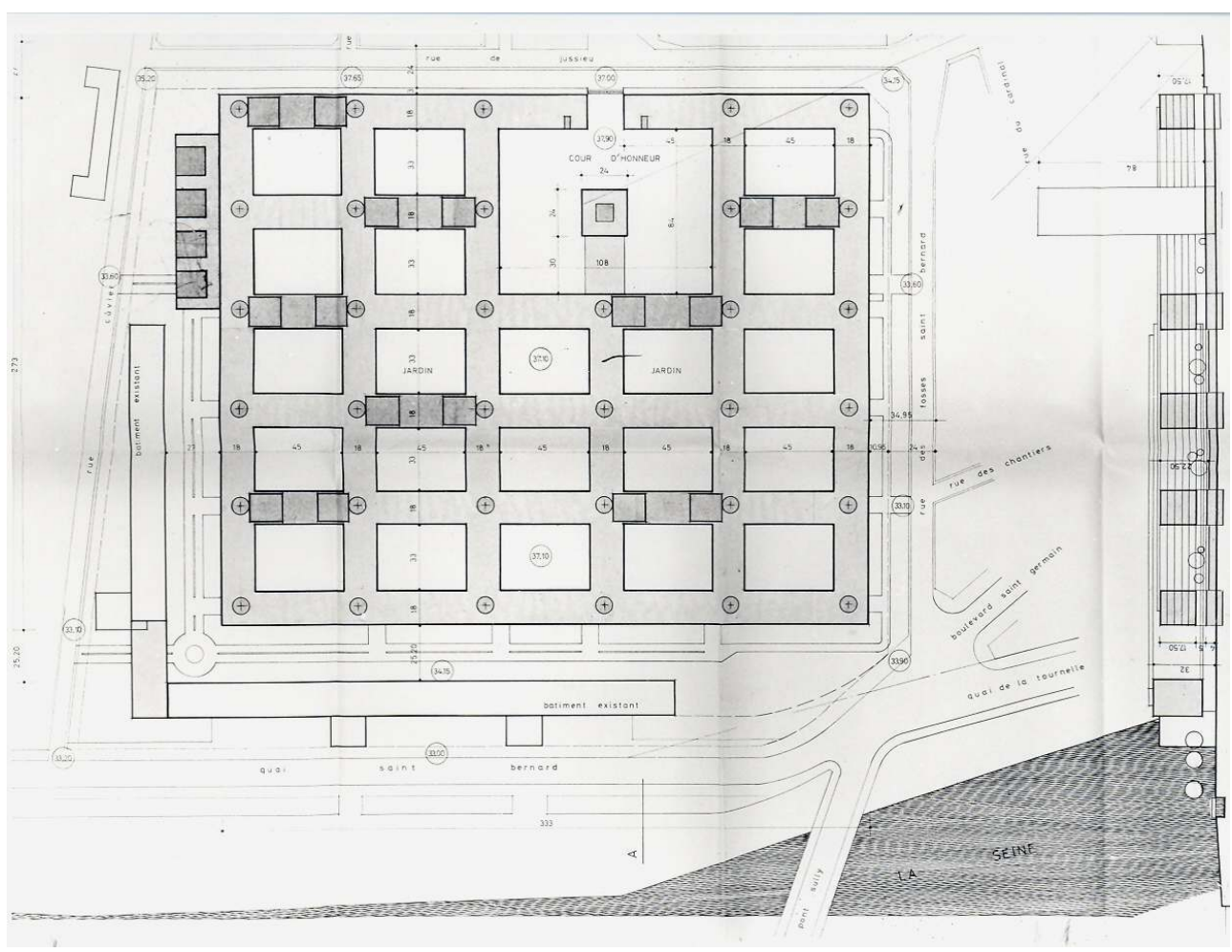

Projet pour la Faculté des sciences de Paris sur le site de la Halle aux vins, plan masse, 1963. Arch. Ville de Paris, 1069 W 124 304222/63

Cl. Jean-Christophe Doërr.

Si Albert conserve dans son plan masse ${ }^{12}$ l'entrée principale au sud, il rompt en revanche avec le principe auparavant dominant de la dispersion d'imposants bâtiments dans un espace arboré. Son plan de masse est un plan massif, puisque sa faculté de quelques 400 $000 \mathrm{~m}^{2}$ se présente comme un vaste quadrilatère de $275 \mathrm{~m}$ par $333 \mathrm{~m}$, percé de vingt et une cours rectangulaires de dimensions identiques, à l'exception de la cour d'honneur, d'une superficie quatre fois supérieure aux autres, au centre de laquelle se dresse la tour d'administration haute de $85 \mathrm{~m}$ qui domine les autres corps de bâtiments, relativement peu élevés $(22,5 \mathrm{~m})$. Cette trame serrée formant un gril peut être décomposée en trois séries d'éléments: grands côtés des cours, petits côtés et tours de contreventement circulaires situées aux intersections ; la réunion de ces trois composantes forme un « $\mathrm{L}$ " qui correspond à une unité d'enseignement et de recherche (la branche courte abritant l'enseignement, la plus longue les laboratoires de recherche). Alors que tous les architectes précédents avaient scindé l'espace disponible en deux niveaux (haut vers la 
place et bas vers la Seine) Albert l'unifie en élevant sa faculté sur une dalle ${ }^{13}$; il crée de ce fait sur l'ensemble du site un double niveau: en bas, les locaux techniques, les circulations et les parcs de stationnement, en haut une vaste dalle piétonne faite de cours formant cloitres et de galeries. L'ossature des bâtiments est entièrement métallique, à base de poteaux de $22 \mathrm{~cm}$ de diamètre et de $4,90 \mathrm{~m}$ de hauteur, disposés en façade tous les $3 \mathrm{~m}$. Chaque poteau supporte une poutre transversale de $18 \mathrm{~m}$ de longueur formant gondole. Au rez-de-chaussée, aucune construction, aucun mur; l'ensemble constitue un système de galeries couvertes totalement ouvert aux vents. À partir du premier étage une trame plus serrée de poteaux (tous les 1,5 m) compose l'ossature des façades, entre eux sont disposés des châssis en acier inoxydable avec allèges en marbre de carrare ${ }^{14}$.

9 Au moment où se dessine dans l'esprit des architectes une physionomie totalement nouvelle pour les bâtiments, l'institution universitaire leur assigne une fonction sensiblement différente de celle envisagée pour les précédents programmes. En effet, dans un premier temps, il a été question de transporter à la Halle aux vins la Faculté des sciences tout entière ; par la suite, et notamment après l'acquisition du domaine d'Orsay, s'est posée la question de la répartition des services entre les deux sites. Le site de la Halle aux vins, avec ses chantiers menés au pas de charge sous la pression d'un accroissement continu des effectifs dans les premiers cycles universitaires, apparaît alors comme un lieu voué à l'enseignement de masse, et non comme un centre de recherche. Cette opposition entre Orsay et Paris est dépassée, au début des années soixante, avec la réflexion que lance le doyen Marc Zamansky. Celui-ci s'appuie sur une vision ambitieuse de ce que doit être une faculté des sciences ${ }^{15}$ : " un immense service public qui fournira [à la Nation] [s]es cadres ${ }^{16}[. .$.$] , et continuera à se consacrer à son rôle fondamental qui est la recherche$ scientifique $»^{17}$. Pour réaliser ce dessein les dirigeants de l'institution envisagent, dans le temps et l'espace, les évolutions de sa physionomie; on évalue la croissance à venir et la répartition par niveaux de la population étudiante, tout en pensant son insertion dans l'espace francilien selon une véritable politique d'aménagement de la région. La traduction de ces prévisions en termes d'implantations universitaires est une structure pyramidale qui confère une place éminente à la Faculté de Paris-centre ${ }^{18}$. En 1970, la Sorbonne totalement abandonnée, l'ensemble de la Halle aux vins doit accueillir 27000 étudiants, dont 25000 en deuxième et troisième cycle et seulement 2000 en premier cycle. Hors de Paris, les sites d'Orsay et de Paris-nord (Villetaneuse, qui est selon Zamansky " une nécessité sociale ${ }^{19}$ ) sont prévus pour recevoir chacun 12000 étudiants répartis dans les trois cycles. Enfin, six collèges scientifiques universitaires (au nombre desquels se trouvent les antennes d'Orléans et de Reims) sont destinés à la formation, en premier cycle uniquement, de 9000 étudiants. Si l'on ajoute que la Faculté de Paris-centre est vue comme le futur siège administratif, on comprend que ce projet hors-normes est bel et bien destiné à devenir, une fois construit, le vaisseau amiral d'une colossale faculté parisienne, forte de plus de 60000 étudiants. Il en sera tout à la fois le centre névralgique et administratif, la tête chercheuse et pensante, le cœur vivant et la vitrine internationale. Enfin, ce projet devrait asseoir la domination des scientifiques sur les littéraires, les juristes et les médecins: aucune autre faculté ne dispose alors d'un programme de développement aussi ambitieux et cohérent.

10 Cette ambition rencontre celle du ministre chargé des affaires culturelles, désireux d'implanter au cœur de Paris une œuvre architecturale majeure. L'intérêt d'André Malraux pour le projet est bien connu : il est à l'origine de l'entrée d'Albert dans le groupe des architectes en charge de l'édifice, il veut, surtout, donner une solennité toute 
particulière à son lancement en présidant personnellement la séance du conseil général des bâtiments de France du 25 avril 1963 consacrée à l'examen du dossier. D'emblée il évoque la relation forte qui doit exister entre l'œuvre à bâtir et les œuvres qu'elle abritera :

M. le ministre rappelle tout d'abord l'importance que le gouvernement attache à la construction de la nouvelle Faculté des sciences sur l'emplacement de la Halle aux vins et tient à souligner l'attention particulière qu'il porte à cette réalisation qui constituera une tentative assez exceptionnelle d'architecture linéaire devant permettre, si elle est acceptée, de confier la décoration de l'édifice à quelques-uns uns des plus grands artistes contemporains ${ }^{20}$.

11 L'intérêt de Malraux pour la Faculté ne se dément pas par la suite, comme en témoignent les nombreuses installations d'œuvres d'art faites dans le cadre du $1 \%$, même s'il faut souligner que les décors monumentaux les plus ambitieux n'ont pas été réalisés (on pense aux œuvres de Braque ou Léger) ${ }^{21}$. L'engouement pour ce "grand chantier» des deux septennats gaulliens semble du reste partagé par d'autres... À la même époque, le quartier de La Défense commence à s'édifier selon un programme rigoureux et empreint de classicisme qui sera ultérieurement abandonné ; il suscite de semblables enthousiasmes, Robert Auzelle n'hésitant pas à citer Baudelaire à son propos: «Le tout est ordre et beauté, luxe, calme et volupté ${ }^{22}$. Une phrase qui dans l'esprit de ses promoteurs, pouvait sans aucun doute s'appliquer parfaitement à la Faculté des Sciences...

\section{Deux universités sur un chantier en panne}

12 À partir du lancement des travaux en février 1964 le chantier avance rapidement, sans que soient pour autant tenus les objectifs fixés initialement par le doyen. Les deux tranches de la physique sont achevées en 1966, puis viennent les services de géologie et de mathématiques (entre 1967 et 1969), tandis que la chimie est réalisée entre 1969 et 1971, en même temps que la tour destinée à l'administration; enfin, on lance la construction des services de biologie, dont la première tranche est terminée en octobre $1972^{23}$. À cette date les principaux inspirateurs du projet ont quitté la scène. Albert est mort brutalement en 1968, Urbain Cassan et René Coulon restent à leur poste, assistés de Constantin de Gortchakoff. Malraux n'est plus ministre depuis 1969, et Marc Zamansky n'est plus doyen, la Faculté des sciences ayant éclaté entre plusieurs universités nouvelles. À la fin de l'année 1972, outre les bâtiments ceinturant la cour d'honneur et la tour, on a entièrement édifié les constructions ceinturant douze cours. Neuf autres, prévues sur le plan initial, n'ont pas été bâties. Au nord manque toute la ligne de bâtiments qui devait faire face à la barre $\mathrm{ABC}$ donnant sur le quai, à l'est seules les constructions entourant la cour la plus méridionale ont été élevées. La disparition des plus fervents initiateurs est sans aucun doute, tout autant que la crise économique, la cause principale de l'arrêt des travaux, ou, plus exactement de leur fort ralentissement, en même temps que s'impose à tous le choix « d'achever » la construction en ne réalisant pas toutes les tranches du projet initial.

13 Ceci étant, il faut songer que la suppression de la Faculté des sciences et la répartition des locaux entre deux universités nouvelles qui s'opposent sur bien des points (et parfois s'affrontent vivement) figurent certainement parmi les raisons, plus ou moins directes, de l'essoufflement du chantier. Ces deux institutions sont issues de la loi d'orientation sur l'enseignement supérieur promulguée le 12 novembre 1968, qui prévoit l'autonomie des universités, et de l'arrêté officiel du 21 mars 1970, qui définit les caractéristiques de 
l'ensemble des universités parisiennes. Entre temps, enseignants et chercheurs issus des anciennes facultés ont rejoint, généralement sur la base d'affinités scientifiques, syndicales ou politiques leurs nouveaux services de rattachement. Sur le site de l'ancienne Faculté des sciences sont désormais présentes les universités Paris VI et Paris VII. Si la première, plutôt conservatrice et centrée autour des sciences et de la médecine, peut être regardée comme la continuatrice la plus légitime de la faculté antérieure, la seconde, née autour d'un projet interdisciplinaire, s'inspire en partie de la récente expérience de Vincennes, se veut novatrice dans tous les domaines et est le lieu d'une intense activité politique ${ }^{24}$. Les tensions politiques entre les deux établissements sont très vives, la simple cohabitation parfois problématique. La question de la répartition des locaux est bien évidemment centrale, et se trouve âprement débattue en 1971 sous l'arbitrage du ministre de l'Éducation nationale. Elle parait aboutir au mois de mars de cette année ${ }^{25}$, mais il semble bien que les décisions rendues n'aient pas été scrupuleusement appliquées, les universités passant entre elles des conventions qui amènent l'une à utiliser certains locaux qui sont la propriété de l'autre, et réciproquement. Les plans d'occupation dressés à différentes époques $\left(1981^{26}\right.$ et $1991^{27}$ notamment) mettent en évidence l'extrême complexité de la division des locaux en fonction des cours et des niveaux: grosso modo, Paris VII tient (en propriété ou en " occupation ") les secteurs situés au nord et à l'est de la cour d'honneur, tandis que Paris VI est implantée vers l'extérieur du site, à l'ouest, au nord (vers la seine) et à la pointe sud-est du bâtiment. Quant à la tour, elle est divisée en deux parties inégales : les onze premiers niveaux pour Paris VII et les vingt-cinq suivants pour Paris VI ${ }^{28}$. Quoi qu'il en soit, par rapport au dessein initial, qui mettait explicitement en lien la monumentalité de l'architecture et l'aménagement du territoire, la situation observable en 1972 est révélatrice d'une perception toute nouvelle de l'espace bâti de "Jussieu»: le site est considéré comme un ensemble de mètres carrés disponibles - en nombre fatalement insuffisant de surcroît ${ }^{29}$ - qui sont susceptibles d'être répartis, au prix de négociations plus ou moins délicates, entre deux établissements aux intérêts divergents. Dans cette perspective, les nouvelles constructions à élever s'inscrivent moins dans la continuation du projet initial que dans la recherche d'une satisfaction immédiate des besoins les plus urgents des universités. Du reste, cette continuité n'est plus justifiée, car elle n'est pas en adéquation avec les fonctions des nouvelles institutions, en particulier celles de Paris VII, désireuse de locaux adaptés aux lettres et sciences humaines (à commencer par des bibliothèques).

Enfin, l'arrêt des travaux rend bien visible une faiblesse pourtant présente dès l'origine dans le projet d'Albert : la problématique liaison entre son gril et les barres construites à la fin des années cinquante par Cassan, Coulon et Madeline. Du fait de l'interruption du chantier, la dalle du gril s'interrompt brutalement, surplombant un vaste terrain vague qui ne va pas tarder à se remplir de constructions parasites, tandis que, vu depuis les barres, le bâtiment d'Albert offre le spectacle piteux de ses pignons dénudés et laisse apparaitre ses substructions de béton qui enferment les niveaux techniques situés sous la dalle (ill. 3). 
III. 3 : Pignon inachevé de la tour de contreventement 12

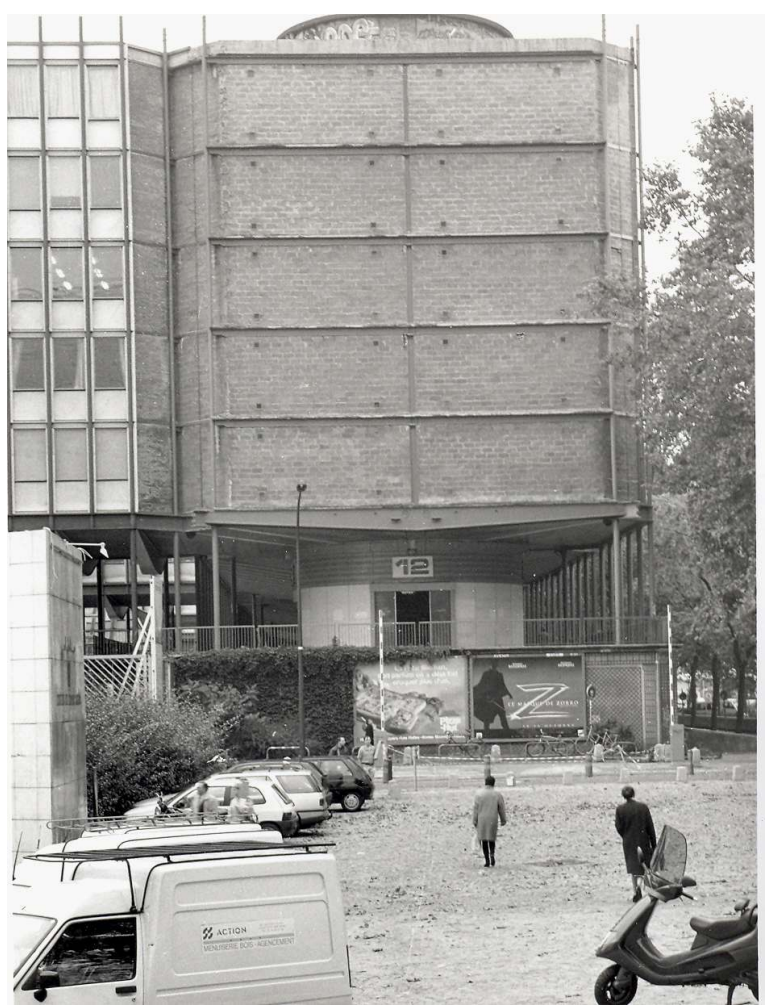

À l'angle de la rue des Fossés-Saint-Bernard et du parvis de l'Institut du monde arabe, hiver 1999.

Cl. Jean-Christophe Doërr.

Tel est l'édifice qui, trente années durant, sera l'objet de projets aussi nombreux qu'éphémères, tandis que peinent à y vivre étudiants et chercheurs et que défenseurs et adversaires de son architecture se combattent avec acharnement.

\section{Trente ans de débats}

\section{Le mal-être des usagers}

Quoi qu'il en soit et bien qu'inachevé, l'établissement actuel - d'ailleurs très visité - est marqué par une grande homogénéité architecturale et est assorti d'œuvres des grands artistes de l'époque. Ceux qui accèdent par l'entrée principale s'ouvrant sur la cour d'honneur ne manqueront pas d'être impressionnés par l'ampleur et les proportions de celleci, ainsi que par les œuvres qui l'entourent : grands pignons décorés par Gischia, mosaique et sols de Lagrange, etc. Or voici que Paris VII a manifesté l'intention d'installer quatre courts de tennis, entourés de grillage et qui occuperont une très importante partie du parvis de la cour d'honneur. Il est superflu d'accompagner de commentaire cette atteinte à un ensemble architectural qui a été minutieusement étudié et sur lequel a longtemps médité un ministre d'État ardent défenseur de l'art ${ }^{30}$.

Commentons pourtant, tant cette lettre irritée paraît bien poser les termes d'une opposition entre architectes et usagers qui se manifeste en ces années par d'autres signes : d'un côté une tentative certes iconoclaste, dans l'esprit du temps, mais visant à répondre à un besoin réel (celui des équipements sportifs) qui n'a pas été pris en compte lors de la conception du bâtiment; de l'autre, avec la conscience de l'inachèvement du 
projet, sa défense et son illustration, étayée par la mise en avant des artistes et du grand homme.

enues ans ce cadre architectural et d'abord elles l'auraient humanisé. Regardez ces couloirs, ces boyaux, ces petites allées, ces cellules, chaque étudiant travaille dans la sienne. La Faculté, ce n'est pas une œuvre d'art ; l'art y est totalement absent, c'est une usine, c'est d'ailleurs assez sinistre. Le Sarcelles de l'Éducation nationale, en mieux tout de même ${ }^{31}$.

19 S'il est besoin de l'humaniser, c'est que Jussieu apparaît dès cette époque comme un lieu « inhumain », au sens où il s'agit d'une architecture qui a été pensée et exécutée sans tenir compte de l'avis des hommes qui vont y vivre, sans envisager la place de l'homme dans son environnement de travail ${ }^{32}$. Ce sentiment semble partagé par tous, du président d'université à l'étudiant de première année, il se manifeste avec force lors du débat organisé le 14 novembre 1972 par Paris VII sur le thème de « l'architecture de Jussieu ». Entièrement retranscrit et enrichi de commentaires, d'annexes et d'articles sur le même sujet, ce débat a été publié dans le journal de l'université, Le Saprophyte, en, janvier $1973^{33}$. Si les articles l'encadrant laissent transparaître les enjeux idéologiques qui sous-tendent cette véhémente critique de l'université «fauriste », le débat entre les architectes et le public, sous la houlette de Michel Alliot son président, est courtois, principalement consacré à rechercher un dépassement à l'incompréhension mutuelle des uns et des autres. Par exemple, René Coulon rappelle avec sa fierté de maître d'œuvre l'enthousiasme des débuts (« C'est sensationnel, construire en plein Paris, dans un terrain qui n'a pas bougé, avec une œuvre à faire, comme ça, pour l'enseignement, la jeunesse et la science. C'est formidable, formidable ! Je vous assure qu'on s'est beaucoup excité sur la question ${ }^{34}$ ) et défend l'essentiel de ce qui a été construit, y compris la tour («On ne peut pas considérer de la même façon des tours de mètres carrés de bureaux commerciaux ou d'habitations et une tour comme celle de la Fac des sciences qui avait dans le paysage parisien une valeur de symbole. C'était un signal comme un clocher, un beffroi, c'était l'Université française $»^{35}$ ). Face à lui, Michel Alliot avance une tout autre idée de l'université, qui subordonne manifestement l'architecture à une fonction toute immatérielle, celle de l'échange: «Pour moi une université c'est d'abord un lieu de rencontre, c'est un lieu de rencontre parce que l'enseignement supérieur se fait comme cela, par un tas de rencontres entre les enseignants et les étudiants. [...] Or j'ai l'impression que cette université a été faite pour éviter les rencontres ${ }^{36}$. Si l'on retrouve là un argument (« la concentration ») proche de celui avancé par Michel Rat, membre de l'Alliance marxiste révolutionnaire, les solutions proposées s'en éloignent, puisqu'en fin de discussion Alliot suggère la création de magasins et de cafétérias dans la cour d'honneur pour «que cela reprenne une allure vivante... Des garçons de café ici, ça changerait beaucoup de choses ", avant de conclure : "Mais là, M. Coulon, vous allez me dire : non, ça gâche mon parti esthétique ». Et de fait, Coulon répond : "Esthétiquement, je suis absolument opposé... Sur ce sujet, on pourrait discuter longtemps $»^{37}$.

20 Au cours des années suivantes, bien loin de s'atténuer, l'insatisfaction des usagers face à ce cadre de vie paraît n'avoir cessé de croître, tout en revêtant de nouvelles formes et un autre ton. Il ne s'agit pas seulement ici d'une évolution politique: le passage de 
l'enthousiasme contestataire des "années de poudre $»^{38}$ au désenchantement ironique des années quatre-vingt-dix s'accomplit au sein d'une université dont les conditions matérielles de travail ne cessent de se dégrader. Ainsi, la critique change d'objet, puisque d'agent d'oppression qu'elle était, l'architecture devient un stigmate, d'abord vécu en silence, avant d'être exposé avec toute l'ostentation que permettent les nouveaux médias. $\mathrm{Au}$ début du XXI ${ }^{\mathrm{e}}$ siècle, deux sites Internet prennent pour thème le délabrement et la saleté du campus de Jussieu : l'un est composé de photographies des endroits les plus sordides du site, assorties de commentaires douceâtres ${ }^{39}$, l'autre est une galerie hallucinante de vues des toilettes les plus sales de l'université ${ }^{40}$. Reste que ces sites ne sont que le reflet déformé, potache et sarcastique d'un problème de sécurité et de santé publique qui est devenu une réalité connue de tous depuis le printemps 1996: le campus de Jussieu est étroitement associé au scandale de l'amiante, et ce plus encore après la déclaration tonitruante de Jacques Chirac, qui, lors de ses vœux du 14 juillet annonce « qu'il n'y aura plus d'étudiants à Jussieu à la fin de l'année ». Les problèmes posés par l'utilisation de l'amiante sont pourtant connus depuis la création en 1974 d'un collectif intersyndical amiante, qui en dénonce les risques, mais c'est en 1994, après la découverte de neuf cas de maladies professionnelles, qu'est créé le comité anti-amiante Jussieu. L'année suivante, les images de la pollution liée à l'amiante sur le campus en font un problème national, tout en liant durablement dans l'esprit du public "Jussieu » et " amiante $»^{41}$. Si l'un des effets inattendus de cette crise réside dans la mise en route d'un plan de rénovation et d'achèvement du site, corollaire de son désamiantage, il n'en est pas moins vrai que, à travers elle, l'architecture de Jussieu n'est plus seulement perçue comme inhumaine, mais bien comme mortelle...

\section{Des défenseurs mal à l'aise...}

21 Devant tant de critiques, et de si rudes, on pourrait penser qu'il ne s'est trouvé personne pour reconnaître des qualités à cette architecture et la défendre envers et contre tout. Depuis le début de la fin, soit depuis 1973, cela est pourtant le cas. Là encore, comme pour les usagers, le discours des défenseurs évolue au fil des ans : proche de l'action et de celui des architectes au commencement, tourné vers la déploration de l'inaboutissement, il se transforme bientôt, revêtant les formes de l'analyse critique et distanciée propre au savoir universitaire. En 1975, lorsqu'il écrit pour la Revue de l'art son texte intitulé « Un grand projet inachevé: la Faculté des sciences de Paris ", Bernard Marrey dresse involontairement - l'acte de décès du dessein d'Albert : il ne sera plus question désormais que de "parachèvement». Surtout, il rappelle l'ambition initiale et consacre de longs développements au programme décoratif et à la tour, dont l'aspect final révèle une trahison des idées d'Albert par ses continuateurs (jaloux ?). Son texte reste une référence essentielle pour les défenseurs futurs.

Moins de dix ans après l'abandon du chantier, alors que le projet pour l'Institut du monde arabe attire de nouveau les regards vers le quai Saint-Bernard, est soutenue une thèse de troisième cycle consacrée au site de Jussieu ${ }^{42}$. Son contenu, bien documenté, reprend des analyses de Bernard Marrey ou de l'article de Philippe Schneyder et Jean Le Chevalier. Cette précoce "objectivation » de Jussieu en annonce d'autres, œuvres d'historiens de l'architecture proches de l'étude du mouvement moderne et de ses continuateurs. Sourd aux critiques de tous ordres (nous sommes en 1998) et ne jugeant que la qualité plastique de l'œuvre, Joseph Abram livre un plaidoyer sans concession: «La rigueur du plan, la 
robustesse du socle, l'évidement du rez-de-chaussée, l'élégance raffinée de l'ossature métallique, la justesse des proportions, la présence des œuvres d'art, font de cet ensemble l'une des meilleures œuvres des années soixante $»^{43}$. Logiquement, cette apologie va de pair avec sa traduction patrimoniale : tel est le sens de la proposition de Gérard Monnier, qui, en réponse aux attaques d'Alain Griotteray ${ }^{44}$, propose non pas de "brûler Jussieu », mais bien, de le classer « sans attendre $»^{45}$, comparant une éventuelle destruction à celle des halles de Baltard.

En énonçant, au-delà du jugement scientifique que légitime sa qualité de spécialiste de l'architecture contemporaine, le caractère patrimonial de Jussieu, Gérard Monnier prend à contre pied les défenseurs du patrimoine traditionnel, pour qui la Faculté est avant tout une atteinte à celui-ci : " Détruire Jussieu, ce serait admettre que la loi de 1913 sur la protection des édifices au nom de l'art et de l'histoire ne s'applique qu'aux édifices antérieurs à 1960, et que, si les instances de l'économie l'exigent, on peut donc raser aussi, entre autres édifices, le musée du Havre, Orly-Sud, et le parc des Princes ${ }^{46}$. En effet, si la tache des défenseurs de Jussieu est malaisée, c'est bien parce qu'ils se trouvent pris entre deux feux: d'un côté les usagers du lieu critiquent son inhumanité et sa dangerosité, restant insensibles aux arguments esthétiques, de l'autre, au nom d'une esthétique antagoniste, les historiens d'art et les spécialistes du patrimoine les plus conservateurs attaquent brutalement le monument.

\section{Un malheur pour le patrimoine?}

Cette forme de critique, déjà présente au cours de la phase active du chantier (notamment à propos de la construction de la tour), n'a jamais cessé. Elle revêt principalement deux formes : d'une part l'architecture de Jussieu est attaquée en raison de ses caractères intrinsèques, d'autre part elle constitue une offense au paysage parisien. La fin des années quatre-vingt-dix est particulièrement riche en critiques sur ces thèmes, avant comme après le scandale de l'amiante. Sans surprise, les continuateurs de l'œuvre de Louis Réau, Guy-Michel Leproux et Michel Fleury, déplorent, photographie judicieusement cadrée à l'appui ${ }^{47}$, la disparition des chais et leur remplacement par cet ensemble «bien représentatif de la médiocrité de l'architecture scolaire et universitaire en France : aucun effort d'adaptation au site, ni d'intégration au quartier, aucun parti original face à la Seine, aucune idée dans l'élévation des façades : un désert spirituel » ${ }^{48}$. Non loin d'eux, Alexandre Gady, qui oublie au passage de citer Albert parmi les architectes, dénonce cet édifice "froid et anguleux $»^{49}$, "une erreur », selon lui, " que seule une destruction totale pourra un jour effacer $»^{50}$. Mais l'attaque la plus violente est celle d'Alain Griotteray, publiée dans Le Figaro juste après l'intervention télévisée du chef de l'État. Voyant dans Jussieu "une des manifestations les plus pitoyables de la déréliction urbaine des années 1960 », il réclame sa destruction : «Jussieu doit disparaître pour en finir avec le martyr imposé depuis près de trente ans à cette partie des quais de la Seine et de l'Ile Saint-Louis. [...] une nouvelle mobilisation s'impose, celle de ceux qui ne se résignent pas à la laideur $~^{51}$. S'il est aux antipodes des plaidoyers propres aux historiens de l'architecture contemporaine, cet argumentaire, tout comme ceux de Michel Fleury et Alexandre Gady, relève toutefois d'une même approche strictement architecturale et, en dernier lieu, patrimoniale, du site: rien n'est dit des difficultés quotidiennes induites par l'architecture de Jussieu. Inconciliables, ces points de vues antagonistes n'en sont pas moins cousins, étant tous deux largement insensibles à la perception de l'œuvre par ses usagers. 
Résumons nous: Jussieu divise autant qu'il est divisé. Voulu comme une expression majeure de l'art contemporain et un symbole de l'unité universitaire (le «beffroi » cher à Coulon), le bâtiment est le lieu de vives tensions internes, et l'objet de non moins vives controverses, ces dernières revêtant une forme complexe, d'une part entre ceux qui " aiment » et ceux qui " n'aiment pas ", d'autre part entre ceux qui « vivent " Jussieu et ceux qui le «voient». L'affaire de l'amiante et ses suites médiatiques confèrent une nouvelle dimension, dramatique, à ces luttes anciennes. De même que son dénouement ultime, on le verra, passe par une solution architecturale, de même, les projets élaborés sans succès entre 1973 et 1993 peuvent être étudiés comme autant de tentatives insuffisantes - pour gommer ces divisions et résoudre ces contradictions.

\section{Vingt ans pour dix projets... dix projets en un an (1972-1992)}

\section{Le S.C.A.P. ${ }^{52}$, les architectes et les universités}

À partir de 1973 et sous l'autorité du service constructeur des académies d'île de France, sont menées un certain nombre de réflexions pour aménager le campus et y édifier de nouvelles constructions. En outre, plusieurs architectes ont mis au point des projets pour le site, apparemment sans avoir reçu pour cela une mission précise de l'administration: on peut y voir le signe d'un intérêt pour le lieu, celui-ci fascinerait les créateurs, tant en raison de son architecture que du fait de son inachèvement. Parmi les architectes concernés, Jean Nouvel occupe une place à part : auteur de l'Institut du monde arabe, implanté à la pointe nord-ouest du site, il ne se contente pas de présenter des projets pour Jussieu ; de fait, son œuvre construite modifie profondément la perception du site et ses possibilités d'aménagement. Pour autant qu'on puisse en juger à la lecture des archives, les schémas ont été élaborés avec la collaboration des deux universités présentes sur le site, à l'exception du " projet Legrand » de 1992, émanation de Paris VI avec une simple «consultation» des instances de Paris VII ${ }^{53}$. Plus que la question des surfaces (en la matière, Jussieu n'est pas le site le plus mal loti en France), le désir contradictoire de rompre l'isolement dont souffrent les universités, tout en mettant fin à leur promiscuité, paraît être le plus petit dénominateur commun de ces réflexions (ill. 4). 
III. 4 : Les universités Paris et VI et Paris VII sur le site de Jussieu

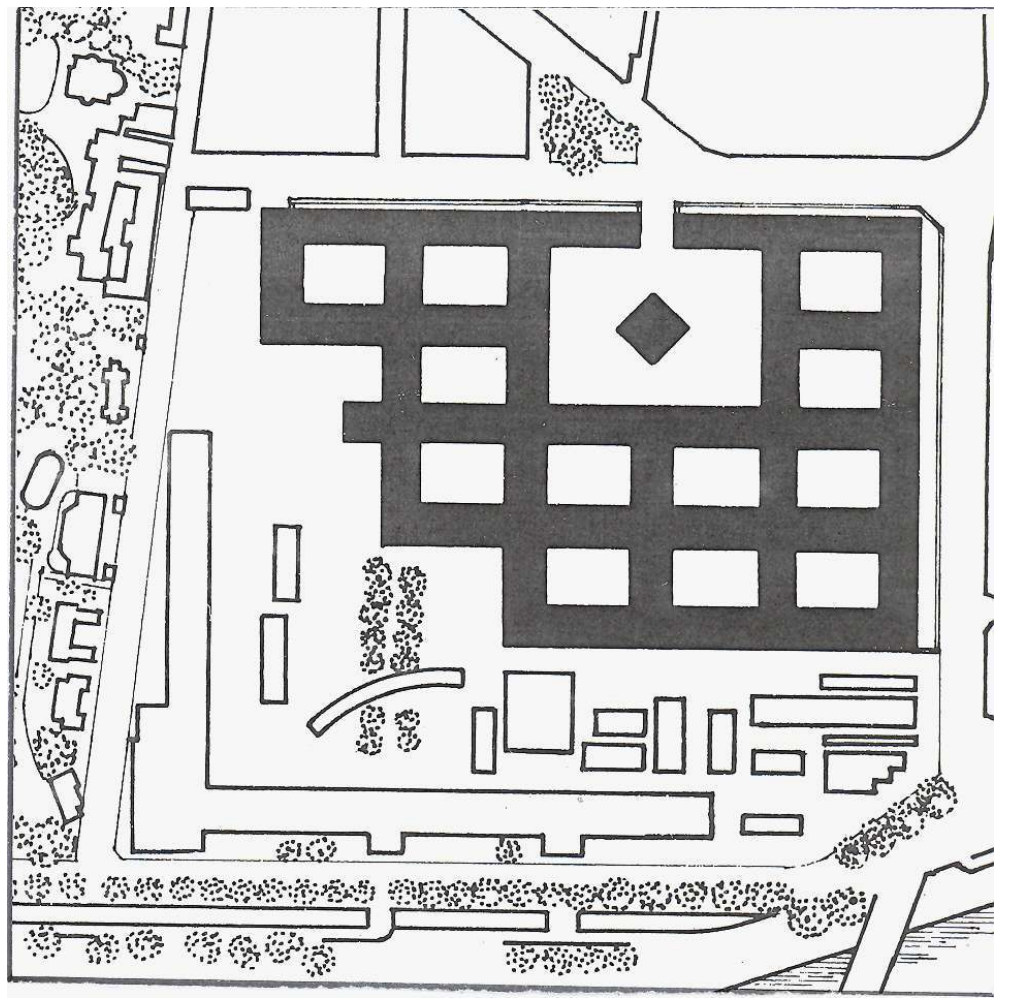

Plan masse, 1983. Arch. de l'Université Paris VII, document non coté

Projets anciens pour Jussieu (1984-1993).

\section{De la continuité à la confrontation}

Après le mort d'Albert, un nouvel architecte, Constantin de Gortchakoff, rejoint René Coulon et Urbain Cassan. En 1973, ils élaborent en fonction des besoins des universités un projet qui repose sur le même principe d'aménagement et les mêmes principes constructifs que celui de 1964. Il en est la version minimaliste : au nord une seule nouvelle cour est implantée, près de l'angle nord-ouest (il s'agit du « grand $\mathrm{U}$ »), tandis que deux autres laissées ouvertes à l'ouest sont fermées par des constructions (on parle de " grand $\mathrm{L}$ » et de " petit $1 »)$. Le terrain compris entre les barres et le gril est traité en jardin paysager, avec «un jeu de courbes et de dénivellations $»^{54}$. Quoique le parti formel du projet s'inscrive dans la continuité de l'existant, les logiques institutionnelles qui le soustendent sont très différentes de celles de la période précédente; il s'agit en effet de remédier à l'enchevêtrement des services et à l'occupation précaire de certains locaux, en permettant aux universités de faire leur "pré carré ». De dures négociations ont alors lieu, Paris VII réclamant une part importante des surfaces à bâtir car elle estime avoir été lésée à plusieurs reprises depuis $1970^{55}$. Faute de crédits, la plus grande partie du programme est abandonnée en $1977^{56}$, une fois réalisés divers aménagements mineurs (une halle des sports sur les deux prévues et le tracé de voiries). En 1982, Constantin de Gortchakoff propose d'habiller de marbre de Carrare les « pignons provisoires » des tours 12 à 42 , laissés à l'état brut après l'arrêt de l'extension. Un tel habillage ferait du provisoire qui dure un état définitif, mais aucune suite n'est donnée à son devis de 3400 000 francs $^{57}$ (ill. 5). 


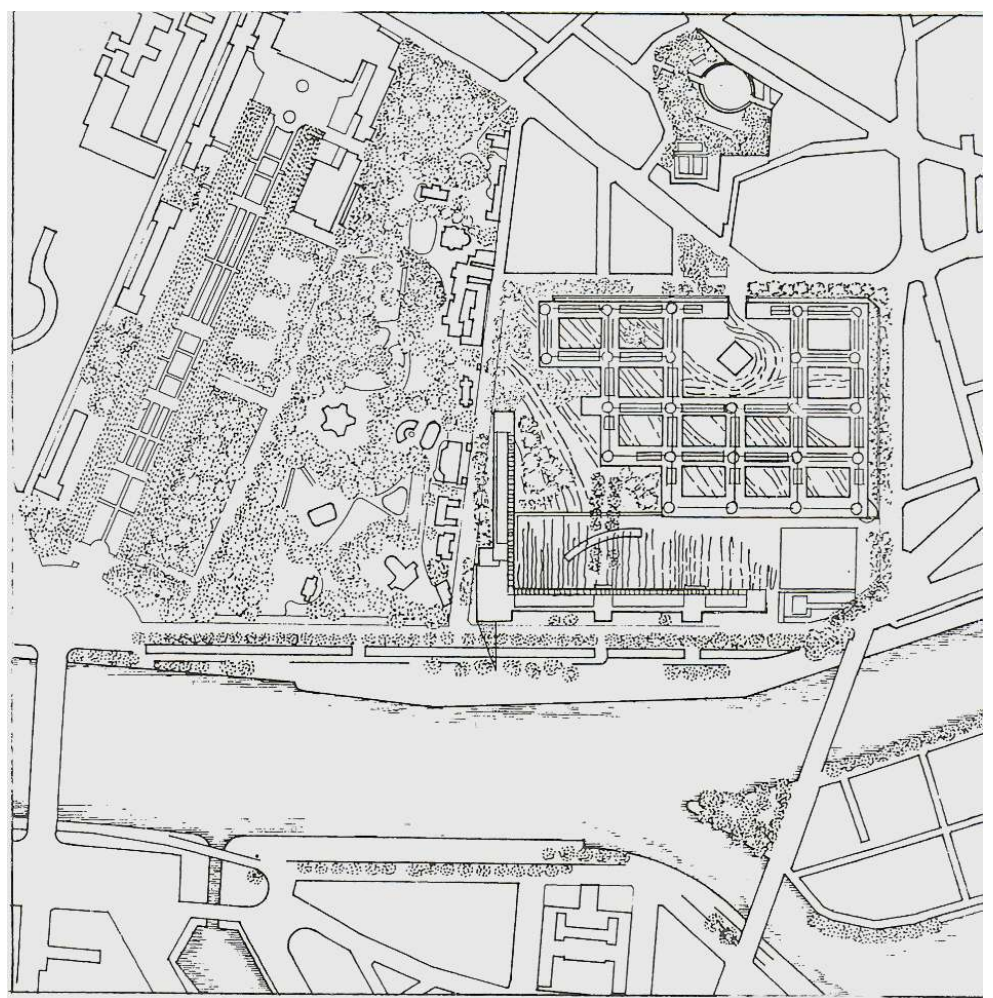

Plan masse, 1983. Arch. de l'Université Paris VII, document non coté

Projets anciens pour Jussieu (1984-1993)

Le concours pour l'édification de l'Institut du monde arabe, fondé en 1980, est remporté par Jean Nouvel, Pierre Soria et Architecture studio. Bien que cet établissement n'ait aucune relation institutionnelle avec les universités, son implantation à l'angle nordouest du quadrilatère, à la place des dernières constructions de la Halle aux vins encore debout, a des conséquences notables sur le devenir de Jussieu. Le bâtiment de Jean Nouvel est de même gabarit que les barres de Cassan voisines et il les prolonge, tout en les masquant à la vue des promeneurs des quais de Seine, mais cet édifice somptueux contraste violemment avec la pauvreté de son environnement, ce qui suscite diverses rancœurs chez les universitaires... Pourtant, là n'est pas l'essentiel : le parvis de l'I.M.A ouvre une brèche dans le site. Pour les aménageurs des projets futurs s'esquisse à cet endroit une nouvelle perspective, une grande avenue ouest-est ; on la retrouvera souvent exprimée dans les projets de la fin des années 1980. Mais tel n'est pas le choix de Jean Nouvel lorsqu'il propose, en 1983, un plan d'aménagement pour l'ensemble de campus. Son propos est beaucoup plus ambitieux, puisqu'il ne s'agit de rien de moins que de l'envahissement de Jussieu par le Jardin des plantes voisin! Il part du constat que le site offre un grand "potentiel", alors que "les abords du bâtiment de la faculté sont un espace de rejet $\aleph^{58}$, dès lors, la rue Cuvier doit disparaître en tant que telle pour être transformée en allée couverte. Au-dessus d'elle, les barrières sont abattues, le jardin se prolonge dans l'université, sur le terrain vague et jusque dans les cours, on crée des reliefs artificiels, comme autant de vagues. Enfin, une passerelle permet aux étudiants d'investir les berges de la Seine. Rien n'est dit ou presque des bâtiments à construire, 
mais Jean Nouvel a posé un diagnostic essentiel: après 1983, toutes les recherches prendront en compte la nécessité d'une ouverture sur le quartier.

Un travail "essentiellement urbanistique ", et la "recherche de tout ce qui contribue à créer un cadre de vie agréable à l'extérieur des bâtiments " ${ }^{59}$ : tel est l'objectif de la mission confiée en janvier 1983 à l'architecte urbaniste Michel Macary. Sans le lyrisme paysager de Jean Nouvel, tel est bien le contenu de sa proposition présentée en octobre 1983. Macary imagine peu de constructions neuves, mais envisage divers aménagements censés créer sur le site des espaces conviviaux : après un escalier monumental, une grande allée plantée, de même niveau que la dalle, assure une continuité entre les barres et le gril, tandis que le parvis de la tour est flanqué de petites constructions inclinées destinées à l'accueil. À l'angle des rues Jussieu et Cuvier, un petit édifice doit abriter des locaux syndicaux et une salle polyvalente, enfin, un pignon nu, orienté à l'est, reçoit un pavillon arrondi prévu pour des espaces culturels $s^{60}$. Bien que relativement modestes, les propositions de Macary ne sont pas suivies d'effets, comme en témoigne la résurgence périodique, dans certains courriers administratifs des mentions de "grand L » ou de " petit 1 » à élever : paradoxalement, le projet de 1973, alors qu'il s'enracine dans une esthétique obsolète et qu'il correspond à un mode d'aménagement de l'espace largement rejeté, est d'une grande longévité dans l'esprit des utilisateurs du campus ${ }^{61} \ldots$.

Il faut attendre la fin des années 1980 pour assister, sous l'impulsion du S.C.A.R.I.F., à la reprise de la réflexion sur l'aménagement du site. Dans un contexte de relance de la construction universitaire et de développement des partenariats avec le monde des entreprises, on s'interroge sur la possibilité d'un "technopôle» à Jussieu ${ }^{62}$. Les propositions issues de la "réflexion Autheman»- du nom de Michel Autheman, architecte conseil chargé de l'étude - en sont le résultat. La partie la moins originale de sa proposition réside dans la construction de huit corps de bâtiments qui viendraient compléter la partie morcelée du gril. Son apport décisif tient à la recherche d'une cohésion renforcée entre les différentes entités du site. Autheman envisage l'aménagement de deux grandes allées est-ouest (parallèle à la Seine) et nord-sud (parallèle à la rue Cuvier), ainsi que la construction de deux bâtiments de grande hauteur. Le premier, relié au bâtiment F par un pont enjambant la rue Jussieu, prend la place de l'ancien P.C.N. ${ }^{63}$ qu'il faudrait donc détruire. Le second, formant une rotonde entre le bâtiment $\mathrm{F}$ et la barre $\mathrm{ABC}$, constitue un vaste belvédère dominant le fleuve. Auparavant, les adjonctions architecturales n'avaient par rapport au traitement urbanistique du site qu'une valeur cosmétique: désormais il paraît admis que l'on ne peut résoudre les difficultés de vie sur le site que par la construction de nouveaux bâtiments (ill. 6 et 7). 
III. 6 : Michel Autheman, Diagnostic et proposition d'aménagement du campus de Jussieu

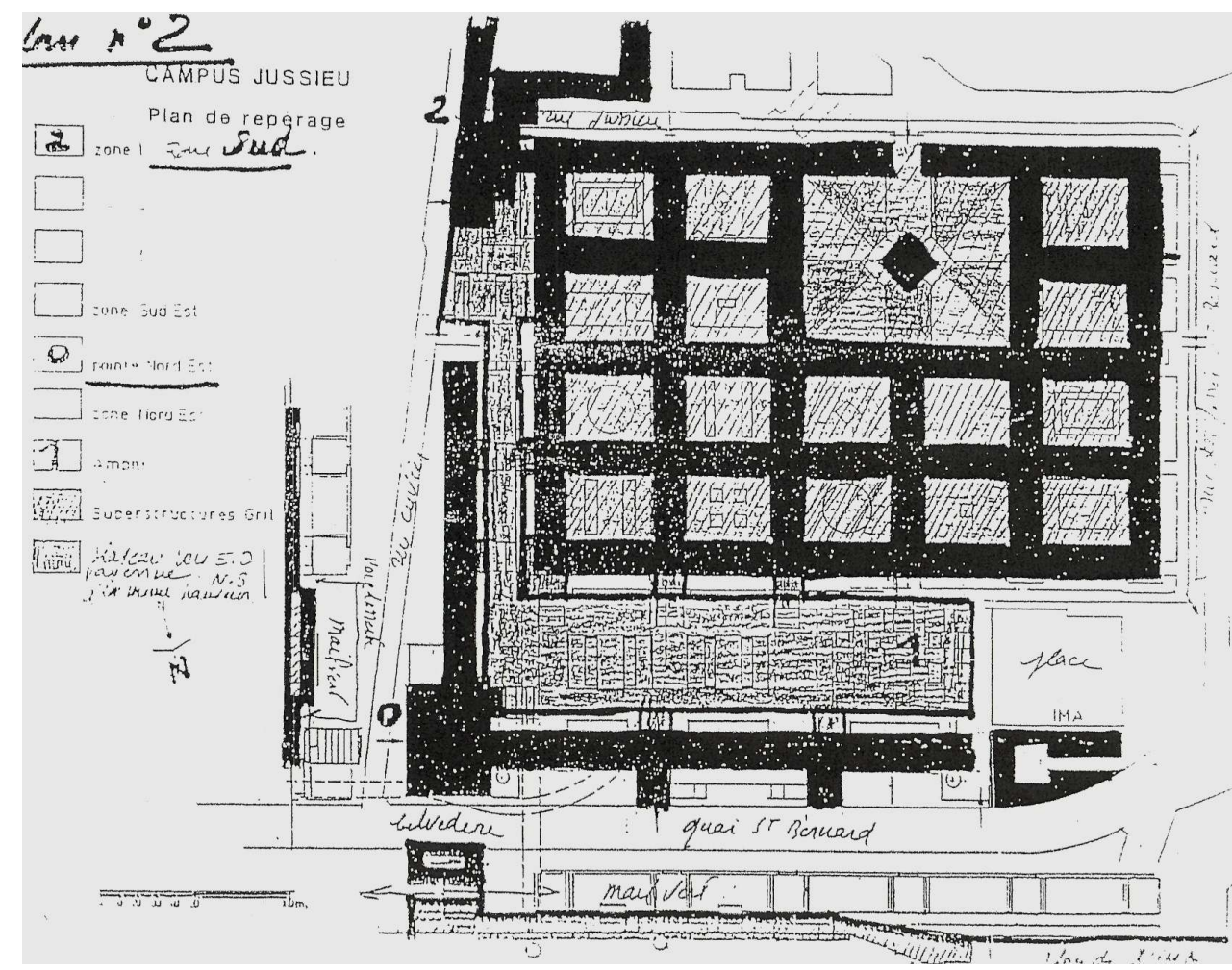

Plan masse, juillet 1988. Concours pour la construction de deux bibliothèques, présentation générale du concours, deuxième cahier (1992)

Archives d'Arlette Pailley-Katz (Université Paris VII), document non coté 


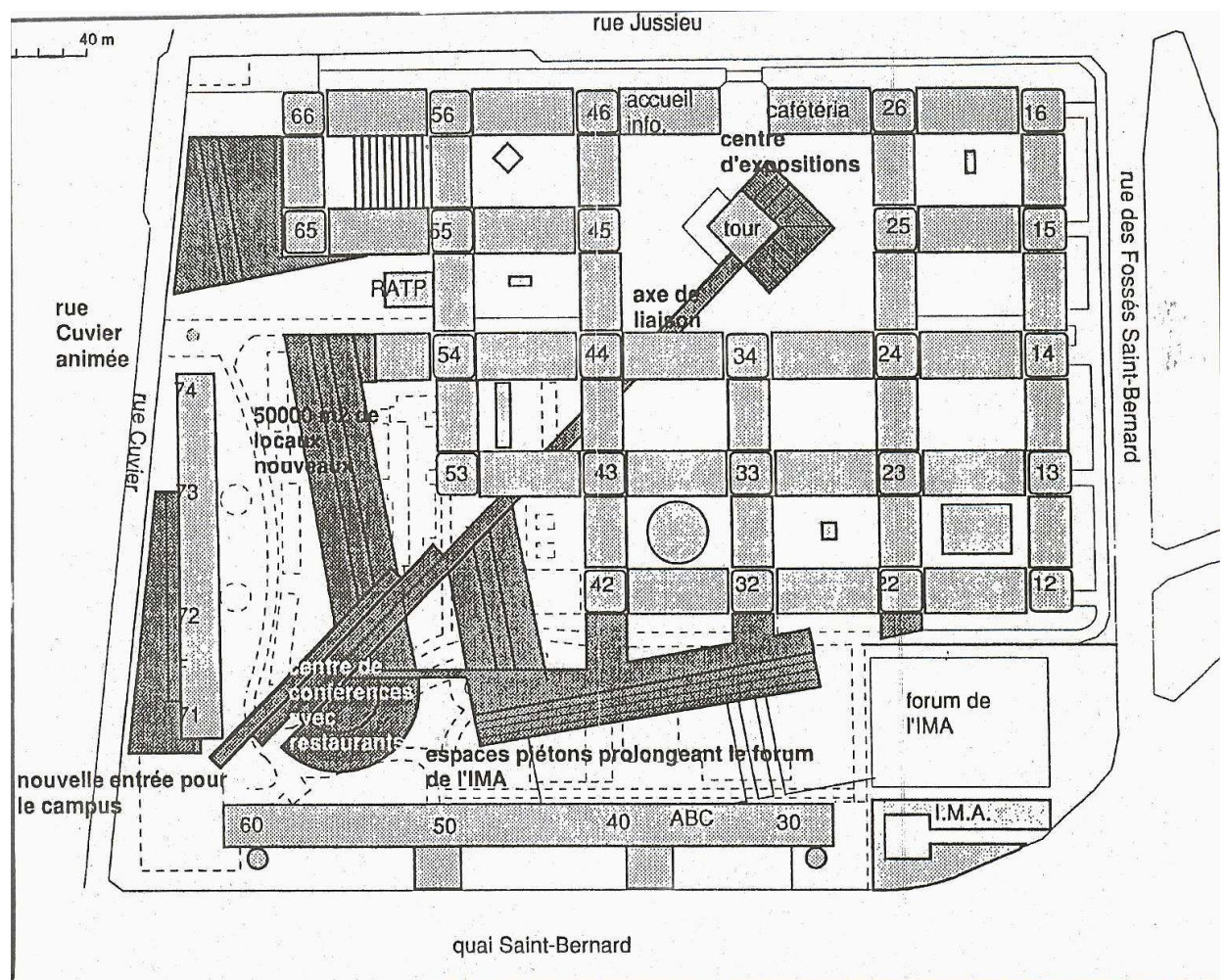

Concours pour la construction de deux bibliothèques, présentation générale du concours, deuxième cahier (1992)

Archives d'Arlette Pailley-Katz (Université Paris VII), document non coté

Tous les projets ultérieurs ${ }^{64}$, ceux de Michel Guy et François Cantal-Duparc en avril 1989, Michel Guy en 1990 et Jean-Claude Legrand en 1992 tiennent compte des acquis de la réflexion Autheman. Michel Guy et François Cantal-Duparc recherchent l'ouverture sur le quartier par la multiplication des entrées, et choisissent de placer la nouvelle porte d'accès principale à l'angle de la rue Cuvier et du quai, détruisant au passage le restaurant. Partant de cet angle, un grand axe de liaison piétonnier traverse l'espace libre et le gril pour aboutir au pied de la tour. $50000 \mathrm{~m}^{2}$ de locaux nouveaux se greffent sur ce cheminement, implantés en diagonale par rapport au damier d'Albert et à l'équerre des barres de Cassan. Ils abritent, comme on peut s'y attendre des locaux destinés à l'accueil du public et aux rencontres : centre de conférences, restaurant, centre d'expositions, etc ${ }^{65}$ . La proposition avancée en 1992 par Jean-Paul Legrand (président de Paris VI) et Mme Abravanel (pour l'Association de sauvegarde du patrimoine historique et artistique de la France) est encore plus ambitieuse: à l'heure d'une collaboration étroite avec les entreprises, ils imaginent pour Jussieu un «centre scientifique international du quartier latin ». L'entrée en est à l'angle de la rue Cuvier et du quai, une longue diagonale mène jusqu'à la tour, trois pavillons de plan carré occupent l'espace entre le bâtiment $A B C$, et le gril, reliant le premier au second par un système de passerelles également disposées en diagonale ${ }^{66} .$. Ce projet s'accompagne d'une prise en compte de la valeur patrimoniale de l'îlot Cuvier, l'ancien P.C.N. qui abrita les laboratoires des Curie, et devrait être restauré. Précisons toutefois que la réflexion développée par l'Université Paris VI en 1992 paraît se faire en dehors des grands schémas directeurs de constructions universitaires. Ses auteurs n'hésitent pas à écrire : «Ce projet ne devrait occulter en rien le plan « Université 
2000 » retenu par le comité interministériel d'aménagement du territoire », une manière comme une autre d'écrire qu'il ne s'inscrit pas dans ce programme.

En tout cas, l'évolution est sensible : il n'est plus question désormais de tourner autour des bâtiments d'Albert ou de Cassan en proposant, ainsi qu'au début des années 1980, le vide des "places", " mails » et autres «esplanades " comme autant de solutions à l'isolement des étudiants et des chercheurs. Si le problème de Jussieu vient de son architecture, c'est de l'architecture que viendra son salut, un salut qui passe par l'édification de grands bâtiments, dotés d'une architecture signifiante, un salut qui appelle de la part des architectes la recherche d'un dialogue avec l'existant, l'affirmation d'une continuité ou celle d'une rupture. Le regard des créateurs se transforme, comme en témoigne la proposition de Jean Nouvel pour les barres de Cassan ${ }^{67}$.

\section{Une bibliothèque, pourquoi ?}

33 En une large vue cavalière de l'histoire culturelle récente, Pierre Nora a défini les années 1980 comme étant le «moment musées », symbolisé par le Grand Louvre, les années 1990 comme le «moment bibliothèques » avec la Bibliothèque nationale de France, en attendant le «moment archives» des années 2000 avec le nouveau centre des Archives nationales à Saint-Denis. C'est bien dans ce contexte général marqué par le grand chantier de Dominique Perrault qu'il faut placer la décision de doter le site de Jussieu d'un grand équipement abritant deux bibliothèques de recherche.

D'un strict point de vue fonctionnel, ce programme correspond à un besoin réel et pressant pour les deux universités, et en particulier pour Paris VII. Au sein de cette dernière, les lettres et sciences humaines sont au départ peu présentes, mais le secteur se structure rapidement et compte bientôt sept U.E.R. (sur les dix-sept de l'Université). Aucun espace n'est prévu pour leur bibliothèque. En 1982, une bibliothèque est aménagée entre les tours 55 et 56 mais elle ne peut se développer que sur $660 \mathrm{~m}^{2}$, ce qui reste très insuffisant ${ }^{68}$. On compte alors une place de lecture pour 270 étudiants ${ }^{69} \mathrm{Il}$ s'agit donc, d'une part, de créer une bibliothèque pour Paris VII ${ }^{70}$, d'une surface de $6500 \mathrm{~m}^{2}$ utiles avec une capacité de stockage de 14,5 kilomètres linéaires et huit cent dix places. D'autre part, les deux universités doivent se rapprocher au sein d'une bibliothèque interuniversitaire scientifique de recherche de $7900 \mathrm{~m}^{2}$ utiles pour 27 kilomètres linéaires et quatre cents places ${ }^{71}$. Enfin, divers espaces de rencontre et une librairie seront des équipements communs installés dans le même bâtiment.

Au-delà de ces impératifs quantitatifs, la décision de construire une bibliothèque s'inscrit pleinement dans la continuité des recherches menées antérieurement par le S.C.A.R.I.F. et les universités pour structurer la vie sur le campus. Plus que les centres de rencontres ou de congrès évoqués dans les projets des années 1980, la bibliothèque est « un espace unitaire, espace d'identification de la communauté universitaire, dans lequel s'inscrit l'ordre des savoirs ${ }^{72}$. Enfin, la bibliothèque marque le retour sur le site de Jussieu d'une implication au plus haut niveau du monde politique. Jack Lang, alors ministre d'État, de l'éducation nationale et de la culture, manifeste un grand intérêt pour la question et suit de près le concours international qui est ouvert. Le parallèle avec l'action d'André Malraux en 1963 est présenté de manière implicite dans le livre publié à l'issu du concours, puisque, à côté de la reproduction des articles de la Revue de l'art et de AcierSteel-Stahl, est donné un extrait du procès-verbal de la séance du Conseil général des bâtiments de France que présida le ministre. Le concours pour la bibliothèque, c'est bel et 
bien la renaissance de Jussieu, grâce à la création d'un grand geste architectural sur ce site exceptionnel mais depuis trop longtemps tombé en déshérence...

\section{Koolhaas, Nouvel et les autres ${ }^{73}$}

Après une première phase de consultation, dix projets sont retenus pour examen par le jury. La force de l'architecture d'Albert impose à tous les concurrents de prendre parti par rapport à elle, soit pour s'inscrire dans son sillage, soit pour rompre avec sa trame ${ }^{74}$. Face à eux, les représentants de la génération précédente donnent également leur avis. Ainsi, Constantin de Gortchakoff tente un temps de s'opposer à toute transformation du gril, qu'il prétend "déposé et protégé ». En revanche, la veuve d'Édouard Albert laisse entière liberté aux architectes, « dans le respect de l'œuvre $»^{75}$. Parfois associés, comme à Euralille où l'un conçoit le schéma d'ensemble tandis que l'autre crée le centre commercial, Rem Koolhaas et Jean Nouvel sont plus souvent rivaux ${ }^{76}$, et cette confrontation de deux esthétiques en annonce d'autres, avec en dernier lieu celle du concours pour la rénovation du forum des $\mathrm{Halles}^{77}$. Leurs projets peuvent servir d'étendards aux deux grandes familles de concurrents en présence. Jean Nouvel, Cruz et Ortiz, Emmanuelle et Laurent Beaudoin font le choix de la continuité, le premier écrivant avec franchise : «Je propose de suivre les lignes d'Albert. Fidèlement, rigoureusement et de vider largement le reste $\aleph^{78}$. Face à eux, emmenés par Rem Koolhaas, cinq équipes ${ }^{79}$ refusent de s'inscrire dans cette linéarité et placent leur bibliothèque entre gril et barres ${ }^{80}$. Koolhaas avec son "gros cube " $^{81}$, " planté » à côté du gril, parie que cette opposition permettra de régénérer les conditions d'existence du lieu. L'équipe de Coop Himmelblau ( Prix, Swiczinsky et Weichenberger) se distingue en proposant un édifice en diagonale, à la structure complexe, fait de deux unités indépendantes réunies par une composition chaotique de verre et d'acier, « structure tridimensionnelle en forme de $\mathrm{X}$ ».

Lors des débats, les rapporteurs des différentes commissions font ressortir les qualités respectives mais antagonistes des projets de Koolhaas et Nouvel ${ }^{82}$. Le jugement rendu donne lieu à de vifs mécontentements. Jean Nouvel est choisi pour l'aménagement d'ensemble du site, ce qui provoque la déception des universitaires, furieux de voir se prolonger l'architecture d'Albert ${ }^{83}$. Rem Koolhaas remporte le concours pour la bibliothèque, et recueille celle des bibliothécaires ${ }^{84}$. Ils critiquent en particulier l'agencement intérieur des salles ${ }^{85}$, où se trouvent $35 \%$ de surfaces présentant des pentes de 0 à 4 ou 6 à $9 \%$ ! Koolhaas livre certes un diagnostic que beaucoup pourraient approuver ("L'implantation de la nouvelle bibliothèque de Jussieu pourrait effacer le déficit social accumulé depuis son achèvement $)^{86}$, mais le remède paraît impossible à administrer. Bien que lauréat, le projet doit être lourdement retravaillé avant d'aborder la phase de construction. Celle-ci tarde à venir. D'abord réduit en surface, le projet est bientôt purement et simplement abandonné. Aucune réserve budgétaire ne semble avoir existé pour financer la construction mais il n'est pas exclu que son caractère éminemment politique lui ait en définitive beaucoup nui, puisque les élections du printemps 1993 entraînent un changement de majorité.

Pour Jussieu, c'est un «enterrement de première classe $»^{87}$. Vingt ans après le premier choc pétrolier qui sonna le glas des ambitions initiales, la réflexion sur l'aménagement du site est au point mort. Le gril demeure inachevé, il se détériore lentement, tout comme les barres de Cassan. Entre ces deux entités, sur le terrain vague que domine la dalle, ont poussé de petites constructions plus ou moins pérennes... dont les bureaux du 
S.C.A.R.I.F.! Le lieu reste cloisonné, construit en opposition avec le quartier environnant, son architecture est mal aimée des usagers. Timidement, on envisage une nouvelle opération, à l'emplacement du bâtiment Esclangon, pour une résidence destinée à l'accueil des étudiants étrangers... Mais, là encore, tout paraît bien incertain ${ }^{88}$. Que faire ?

\section{LES TRIBULATIONS ARCHITECTURALES DE L'UNIVERSITÉ FRANÇAISE : JUSSIEU, SUITE ET FIN ? (1992-2007)} pas un cas isolé dans l'histoire de l'architecture universitaire française. Bien au contra La complexité du dossier, évidente à la lecture de cette étude, en fait plutôt un cas d'école. Il faudrait ici revenir de manière approfondie sur plusieurs exemples de grands projets architecturaux destinés aux institutions universitaires qui ne virent jamais le jour ou connurent une exécution si lente que le résultat bâti ne pouvait plus être qu'en inadéquation avec les besoins des établissements. De l'Université impériale de Poyet à Jussieu, en passant par les nombreuses tentatives de reconstruction de la Sorbonne et l'affaire calamiteuse de la nouvelle Faculté de médecine, les cas ne manquent pas, qui mériteraient d'être détaillés. Plans par dizaines jamais suivis d'exécution, projets grandioses qui rétrécissent à force de chantiers languissants et de politiques d'aménagement erratiques, bâtiments venus trop tard et construits pour les générations antérieures : jusqu'aux offenses faites au ciel de Paris au nom de la science, tout cela se retrouve à Jussieu. Sauf peut-être ce qui constitue le dernier acte de cette histoire, d'autant moins aisé à écrire pour l'historien qu'il s'y trouve contraint au journalisme et à l'observation directe, faute d'archives accessibles.

1996 - mais depuis combien de temps ne s'en doutait-on pas? - une histoire d'architecture universitaire et d'aménagement urbain rejoint un problème majeur de santé publique qui devient bientôt un scandale d'État. Comme l'a montré Sonya Bertrand, la publication du rapport de l'I.N.S.E.R.M. concernant les effets de l'amiante sur la santé intervient au moment où est déjà organisé sur le site un « collectif amiante ». Alors que les retombées du rapport constituent un fait majeur de l'actualité au printemps et durant l'été 1996, l'affaire de Jussieu, entretenue par les annonces de déménagements en catastrophe, prend la forme d'un feuilleton durable, ponctué d'articles fréquents, mais plus brefs que ceux traitant du rapport ${ }^{89}$. Au-delà du déménagement et des risques encourus par les populations vivant sur le campus, la question de sa démolition est posée... Mais peut-on supprimer une des plus importantes concentrations de scientifiques en France et laisser sans locaux des établissements dotés d'une renommée internationale établie?

Le désamiantage semble en définitive une solution plus appropriée ${ }^{90}$, et elle trouve un début de concrétisation le 17 janvier 1997 avec la création de l'Établissement public du campus de Jussieu. Outre l'assainissement des locaux, cette nouvelle structure est en charge de toutes les opérations devant permettre la continuité de la vie sur le site, depuis le relogement provisoire des unités jusqu'à la rénovation des édifices. À la même époque, le programme de construction U3M, dont une des priorités est le retour des universités dans les centres urbains, permet à l'Université Paris VII d'envisager une nouvelle implantation pour tous ses services ${ }^{91}$. La partie méridionale de la Z.A.C. de Paris - rive gauche en est le site, les vestiges des grands moulins de Paris en sont les bâtiments phare, ceux qui abritent la bibliothèque qui a si longtemps fait défaut à l'université. 
Quant au site de Jussieu, désormais dévolu à la seule université Paris $\mathrm{VI}^{92}$, il fait l'objet d'un programme d'aménagement complexe, protéiforme et encore peu lisible à ce jour, alors que de nombreuses constructions provisoires liées au désamiantage occupent les espaces libres. Un tel pullulement n'est pas dépourvu de charme, mais parait laisser entier le problème jamais résolu de la relation entre le gril, objet de toutes les attentions, et les constructions antérieures, encore délaissées. Le campus est d'abord travaillé sur sa périphérie, avant que ne soit traité son fonctionnement interne. Deux bâtiments à usage d'enseignement, l'un de type industriel ${ }^{93}$, l'autre apparaissant comme un geste architectural de prestige ${ }^{94}$, s'accrochent aux barres inachevées à l'est. Sur la façade nord, les pignons laissés nus sont flanqués de pavillons, sans doute inspirés de ceux de Nouvel pour le concours de 1992, qui abritent des résidences pour chercheurs et étudiants ${ }^{95}$. Enfin, les opérations de rénovation du secteur ouest prévoient la création de deux axes de circulation se coupant à angle droit, l'un d'entre eux prenant naissance au niveau de la rue des Fossés Saint-Bernard et gagnant le niveau de la dalle par un long plan incliné, tandis que l'autre, sous la forme d'une rue intérieure fermée, accueillerait notamment les services de la scolarité et de la vie étudiante. Deux nouvelles bibliothèques seraient les principaux équipements à créer dans ce secteur. Faut-il s'en étonner? Un groupement d'architectes qui a écrit quelques-unes des grandes pages de la réhabilitation des châteaux industriels, Reichen et Robert, est le maître d'œuvre de cette opération. Plus que d'une rénovation, il s'agit d'une transformation radicale, trente ans après l'abandon du chantier. Sur ce point du moins, Zamansky avait raison, une faculté des sciences se construit bien pour trente ans...

\section{NOTES}

1. Marc Zamansky, "Une Faculté des sciences se construit en trois ans pour trente ans", Éducation nationale, $\mathrm{n}^{\circ}$ 34, 30 novembre 1961, p. 5-7, p. 7.

2. Xavier Laurent, «Les sept merveilles de la France : la loi de programme du 31 juillet 1962 sur les Monuments historiques", Livraisons d'histoire de l'architecture, no 3, 1er semestre 2002, p. 113-125. Les monuments en question sont Fontainebleau, Versailles, Vincennes, le Louvre, les Invalides, Chambord, et la cathédrale de Reims.

3. On fait remonter au Second Empire les premiers projets, attribués à Haussmann, pour l'installation de la Faculté des sciences sur le site de la Halle aux vins. Louis Liard évoque cette idée dans son ouvrage : Louis Liard, L'Université de Paris, Paris, Armand Colin, 1909, 2 vol., vol. 1, 132 p., p. 97.

4. Sur le projet lui-même, voir l'article de Bernard Marrey: «Un grand projet inachevé : la Faculté des sciences de Paris ", Revue de l'art, no 29, 1975, repris dans Campus universitaire de Jussieu, naissance d'une grande bibliothèque, Paris, Sens et Tonka, 1993, 251 p., p. 32-51.

5. Voir Christian Hottin, «La nouvelle Sorbonne ou l'impossible défi de M. Nénot », La Sorbonne: un musée, ses chefs d'œuvre, Paris, chancellerie des Universités de Paris - RMN, 2007, 280 p., p. 74-97. Une version provisoire et non illustrée de ce texte est déposée sur le serveur HAL-SHS : [notice halshs-00121241. 
6. Les archives de l'Université Paris VII son généralement identifiées sur le dos du carton par un résumé de son contenu: "parachèvement » est l'expression la plus couramment usitée pour désigner toutes les affaires liées à la construction de nouveaux bâtiments sur le site de Jussieu.Ni 7. Il s'agit du bâtiment Félix Esclangon. Voir «L'inauguration des laboratoires Félix Esclangon » Annales de l'Université de Paris, $27^{\mathrm{e}}$ année, $\mathrm{n}^{\circ}$ 2, avril-juin 1957, p. 204-205. La construction de cet édifice a d'abord été étudiée par le Conseil général des bâtiments de France : Arch. nat. (C.H.A.N.), F 216673.

8. Commencés en 1958, il seront achevés en 1961. Voir Arch. nat. (C.H.A.N.), F ${ }^{21}$ 6673. Il s'agit des bâtiments $A B C$ (sur le quai) et $F$ (sur la rue), toujours en service aujourd'hui.

9. Arch. nat. (C.H.A.N.), $\mathrm{F}^{21}$ 6673. Faculté des sciences, étude de plan d'ensemble.

10. Arch. nat. (C.H.A.N.), $\mathrm{F}^{21}$ 6673. Université de Paris, nouvelle Faculté des sciences, étude de plan d'ensemble.

11. Bernard Marrey, op. cit. , p. 35.

12. Pour des descriptions complètes du projet architectural, voir, outre l'article cité de Bernard Marrey, Philippe Schneyderet Jean le Chevalier, «Moderne Escurial : la Faculté des sciences de Paris-centre, la plus grande d'Europe », Acier-Steel-Stahl, mai 1967, n 5, p. 209-219, repris dans Campus universitaire de Jussieu, naissance d'une grande bibliothèque, Paris, Sens et Tonka, 1993, 251 p., p. 16-31.

13. Sur le principe de la dalle, voir Virginie Lefebvre, Paris - Ville moderne, Maine-Montparnasse et La Défense (1950-1975), Paris, Norma, 2003, 326 p., p. 185-188.

14. Bernard Marrey, op. cit., p. 38-39.

15. Cette vision s'appuie sur les travaux d'économistes, notamment ceux de Jean Fourastié. Le IV Plan de modernisation de la France prévoit le doublement du niveau de vie en quinze ans, entre 1960 et 1975. Cela suppose une croissance comparable du nombre d'ingénieurs, de cadres, de chercheurs, de techniciens et d'enseignants. Roger Poullain, «La rentrée 1964 à la Faculté des sciences ", Éducation nationale, 26 janvier 1961, nº 4, p. 8-10.

16. On reconnaît là la perception - et la construction - par les élites de cette nouvelle catégorie sociale, en partie sous l'influence du modèle américain. Voir Luc Boltanski, Les cadres, la formation d'un groupe social, Paris, Minuit, 1982, 523 p. Voir plus particulièrement : «Les universités et les entreprises » (p. 354-358) et « La modernisation de l'université (p. 358-364).

17. Marc Zamansky, op. cit., p. 6.

18. Arch. nat. (C.H.A.N.), $\mathrm{F}^{21}$ 6673. Programme pédagogique de Marc Zamansky pour la Faculté des sciences [1962].

19. Arch. nat. (C.H.A.N.), ${ }^{21}$ 6673. Programme pédagogique de Marc Zamansky pour la Faculté des sciences [1962].

20. Arch. nat. (C.H.A.N.), $\mathrm{F}^{21}$ 6673. Procès-verbal de la séance du 25 avril 1963 du Conseil général des bâtiments de France. Repris partiellement dans : Campus universitaire de Jussieu, naissance d'une grande bibliothèque, Paris, Sens et Tonka, 1993, 251 p., p. 13-15, p. 13.

21. Sur les décors de Jussieu, voir Bernard Marrey, op. cit., p. 39-46.

22. Robert Auzelle, dans les Annales de l'industrie du B.T.P. en novembre 1961. Cité dans Dominique Oboussier, Le Centre Jussieu : étude architecturale et urbanistique ou la recherche de l'architecture de la Faculté des sciences de Paris de la monarchie de Juillet à nos jours, thèse de doctorat de troisième cycle sous la direction de Marc Le Bot, Université Paris I - Panthéon Sorbonne U.F.R d'art et d'archéologie, 1982, 288 ff. dact., p. 70.

23. Institut français d'architecture (I.F.A.), 133 IFA - 2/7. Dossiers de la direction de l'architecture et du patrimoine, dossier Édouard Albert, p. X.

24. Sur la fondation de Paris VII, voir Marie-Claire Hoock-Demarle, La Galaxie Diderot, les lettres et les sciences humaines à Paris 7 - Denis Diderot, Paris, Syllepse, 2004, 286 p., p. 25-54.

25. Voir la lettre du ministre de l'éducation nationale du recteur de l'Académie de Paris, datée du 18 mars 1971. Arch. du rectorat, S.C.A.R.I.F., archives du site de Jussieu, boite n 8. 
26. Arch. de l'Université Paris VII, boite $n^{\circ}$ 53-9. Répartition des locaux des deux universités en 1981.

27. Arch. de l'Université Paris VII, boite n $^{\circ}$ 53-11. Répartition des locaux de l'Université Paris VII en 1991.

28. Arch. de l'Université Paris VII, boite $n^{\circ}$ 53-9. Répartition des locaux des deux universités en 1981.

29. La Faculté, entièrement construite, était prévue pour l'accueil de 27000 étudiants. Dans les années quatre-vingt, la population du site dépasse les 40000 puis 50000 personnes.

30. Arch. nat. (C.H.A.N.), ${ }^{21}$ 6673. Lettre de l'architecte Urbain Cassan au recteur de l'Académie de Paris, datée du 7 juillet 1971.

31. Entretien avec un chercheur au laboratoire de zoologie, cité dans: Art et architectures. Bilan et problèmes $d u 1 \%$, ministère d'État aux affaires culturelles - centre national d'art contemporain, Paris, 1970, n. p. Nous remercions Olivier Azzola, responsable des archives de Paris VII, pour la communication de ce document.

32. Cette inadéquation de l'œuvre à l'usager est parfois exprimée sur un mode mineur à travers un phénomène rumoral : il s'agirait alors d'une architecture prévue pour d'autres hommes sous d'autres cieux. Jussieu partage avec d'autres universités (comme celle du Mirail à Toulouse) cette rumeur qui en fait un édifice conçu pour l'Afrique du nord (d'où les toits plats) et que l'on aurait construit finalement en France, après la décolonisation, sans songer à l'adapter au climat francilien.

33. «L'architecture de Jussieu », Le Saprophyte, le journal de Paris VII, janvier 1973, p. 5-10. Nous remercions Oliver Azzola, responsable des archives de Paris VII, pour la communication de ce document tout à fait exceptionnel.

34. «L'architecture de Jussieu », op. cit., p. 6.

35. «L'architecture de Jussieu », op. cit., p. 7.

36. «L'architecture de Jussieu », op. cit., p. 5.

37. «L'architecture de Jussieu », op. cit., p. 10.

38. Hervé Hamon et Patrick Rotman, Génération, t.2, Les années de poudre, Paris, Seuil, 1988, 701 p.

39. http://jussieu.golgothe.net/

40. http://chiottes.jussieu.free.fr/

41. Voir Sonya Bertrand, Le traitement médiatique du rapport de l'I.N.S.E.R.M. sur les effets de l'amiante sur la santé, mémoire de maîtrise sous la direction de Jean-Michel Forestier et Paul Janiaud, U.F.R. de communication, cinéma et information, Université Paris VII, 1997: http:// www.infoscience.fr/travaux/amiante/sommaire

42. Dominique Oboussier, Le Centre Jussieu : étude architecturale et urbanistique ou la recherche de l'architecture de la Faculté des sciences de Paris de la Monarchie de juillet à nos jours, thèse de doctorat de troisième cycle sous la direction de Marc le Bot, Université Paris I - Panthéon Sorbonne U.F.R d'art et d'archéologie, 1982, 288 ff. dact.

43. Joseph Abram, L'Architecture moderne en France, t. 2, Du chaos à la croissance (Gérard Monnier, dir.), Paris, Picard,1999, 328 p., p. 190.

44. Alain Griotteray, « Pourquoi il faut raser Jussieu », Le Figaro, 18 juillet 1996, p. 25.

45. Gérard Monnier, « Ne brûlez pas Jussieu ? [sic] », Libération, 23 août 1996, p. 4.

46. Gérard Monnier, ibid.

47. Louis Réau, Histoire du vandalisme, les monuments détruits de l'art français, édition revue et augmentée par Michel Fleury et Guy-Michel Leproux, Paris, Robert Laffont, 1994, 1190 p., p. 949. En dessous d'une émouvante photographie des chais aux noms pittoresques, la vue en contreplongée d'une des barres de Cassan, et non du gril d'Albert, sert effectivement de repoussoir.

48. Louis Réau, op. cit., p. 949.

49. Alexandre Gady, La Montagne Sainte-Geneviève et le quartier Latin, guide historique et architectural, Paris, Hoëbeke, 1998, 325 p., p. 222. 
50. Alexandre Gady, op. cit., p. 33.

51. Alain Griotteray, op. cit., p. 25.

52. Service constructeur de l'Académie de Paris crée par l'arrêté du 8 juin 1965. Ultérieurement dénommé S.C.A.R.P., il devient le S.C.A.R.I.F. (pour « académies d'Ile de France ») par arrêté du 8 mai 1981.

53. Arch. de l'Université Paris VII, fonds Pierre Barrat, boite $\mathrm{n}^{\circ} 15$. Le «Centre scientifique international du quartier Latin », projet d'achèvement du campus universitaire Jussieu (Pariscentre).

54. Concours pour la construction de deux bibliothèques, présentation générale du concours, deuxième cahier, p. 32. Document transmis par Mme Arlette Pailley-Katz et Mme Catherine Tresson. Je tiens à les remercier pour la communication de cette documentation.

55. Arch. de l'Université Paris VII, fonds de la présidence, document non coté («travaux 1976-1980 et constructions 1975-1980»). Lettre du président de l'Université Paris VII au secrétaire d'État aux universités, datée du 19 juillet 1974.

56. Arch. de l'Université Paris VII, fonds de la vice-présidence chargée des locaux, boite $n^{\circ} 30$. Copie d'une lettre du président de l'Université Paris VI au recteur de l'Académie de Paris, avril 1982.

57. Arch. du Rectorat, S.C.A.R.I.F., archives du site de Jussieu, boite $\mathrm{n}^{\circ}$ 13. Lettre de Constantin de Gortchakoff au directeur du S.C.A.R.I.F., datée du 10 mars 1982.

58. Arch. de l'Université Paris VII, document non coté. Projets anciens pour Jussieu (1984-1993).

59. Arch. de l'Université Paris VII, fonds de la vice-présidence chargée des locaux, boite $\mathrm{n}^{\circ} 30$. Parachèvement du campus de Jussieu, compte rendu de la réunion du 14 décembre 1982.

60. La décoration de sa façade serait confiée à un artiste pour un budget maximal de 400000 francs. Arch. de l'Université Paris VII, fonds de la vice-présidence chargée des locaux, boite $\mathrm{n}^{\circ} 32$. Projet de consultation d'artistes pour l'aménagement d'un pignon du campus de Jussieu, juillet 1984.

61. Arch. de l'Université Paris VII, fonds Pierre Barrat, boite $n^{\circ} 15$. Projet de construction de 20 $000 \mathrm{~m}^{2}$ sur le campus de Jussieu, 19 octobre 1988.

62. Arch. de l'Université Paris VII, locaux et cadre de vie, aménagement du campus (1989-1991), boite $\mathrm{n}^{\circ}$ 576. Lettre du directeur du S.C.A.R.I.F. J.-P. Grunspan à la présidente de l'Université Paris VII et note jointe, datées du 24 mars 1988.

63. Il s'agit du bâtiment destiné à la Physique, la chimie et les sciences naturelles construit à l'angle des rues Jussieu et Cuvier par Nénot. Les époux Curie y installèrent leur laboratoire.

64. Il faut citer pour mémoire le projet de D. et A. Sloan, présenté en mars 1989, mais qui ne semble se rattacher à aucune réflexion institutionnelle: un grand bâtiment courbe, de même hauteur que les constructions du gril, en épouse toutes les infractuosités, depuis le parvis de l'I.M.A. jusqu'à l'angle des rues Jussieu et Cuvier. Arch. de l'Université Paris VII, locaux et cadre de vie, aménagement du campus (1989-1991), boite $\mathrm{n}^{\circ} 576$.

65. Concours pour la construction de deux bibliothèques, présentation générale du concours, deuxième cahier, p. 38.

66. Arch. de l'Université Paris VII, fonds Pierre Barrat, boite $n^{\circ} 15$. Campus de Jussieu, projet de centre scientifique international quartier Latin, avril 1992.

67. Jean Nouvel veut faire émerger la «symbolique navale " cryptique de ces constructions en révélant, à l'aide de superstructures, leur silhouette de porte-avions. Marc Javoy, op. cit., p. 63. Les planches du projet de Jean Nouvel sont présentées en illustration du texte de Marc Javoy. Voir aussi Patrice Goulet, Jean Nouvel, éditions du regard, Paris, 1994, 262 p., p. 42-43.

68. Entretien avec Arlette Pailley-Katz et Catherine Tresson, novembre 2006.

69. Isabelle Crosnier et Isabelle Radlak, «Du concours à la fabrication du projet», Campus universitaire de Jussieu, naissance d'une grande bibliothèque, Paris, Sens et Tonka, 1993, 251 p., p. 104-112, p. 109. 
70. En 1989 on pense installer une bibliothèque plus vaste $\left(1500 \mathrm{~m}^{2}\right)$ dans la barre $54-55$, mais l'idée fait long feu. Entretien avec Arlette Pailley-Katz et Catherine Tresson, novembre 2006.

71. Isabelle Crosnier et Isabelle Radlak, op. cit., p. 110-112.

72. Isabelle Crosnier et Isabelle Radlak, op. cit., p. 105.

73. Les professions de foi des candidats et les documents de présentation sont reproduits dans l'ouvrage Campus universitaire de Jussieu, naissance d'une grande bibliothèque (Paris, Sens et Tonka, 1993, 251 p.) aux pages 126 à 243.

74. Pour une synthèse sur les projets: Jean Attali, «Babel on site, remarques sur dix projets d'architecture ", Campus universitaire de Jussieu, naissance d'une grande bibliothèque, Paris, Sens et Tonka, 1993, 251 p., p. 118-125.

75. Mme Albert juge cette liberté préférable à des interdictions, « l'œuvre de son mari ayant déjà été largement bafouée par la réalisation de la tour centrale». Arch. de l'Université Paris VII, boite $\mathrm{n}^{\circ} 58$ - 2. Campus de Jussieu, concours pour la construction de deux bibliothèques universitaires, procès-verbal du jury (19 et 24 novembre 1992), daté du 4 février 1993

76. Sur les rapports entre ces deux architectes, voir Marco Tabet, La Terrifiante beauté de la beauté, naturalisme et abstraction dans l'architecture de Jean Nouvel et Rem Koolhaas, Paris, Sens et Tonka, 1996, $117 \mathrm{p}$.

77. Françoise Fromonot, La Campagne des halles, les nouveaux malheurs de Paris, La fabrique éditions, Paris, 2005, 156 p.

78. Jean Nouvel, «Le vide est une vertu ", Campus universitaire de Jussieu, naissance d'une grande bibliothèque, Paris, Sens et Tonka, 1993, 251 p., p.144-162, p. 144. Comme au temps d'Albert, une citation baudelairienne s'impose en exergue de son texte : «Je hais le mouvement qui déplace les lignes... ».

79. Outre OMA - Rem Koolhaas, il s'agit de Jacques Hondelatte, Pierre du Besset associé à Dominique Lyon, Toyo Ito et Coop Himmelblau.

80. Enfin, deux équipes récusent cette alternative. Herzog et de Meuron ferment au sud et à l'ouest l'espace libre avec deux bibliothèques distinctes, donnant naissance à une grande cour intérieure traitée en jardin. Architecture studio tourne le dos au gril et préfère investir la base de la barre donnant sur le quai pour offrir aux lecteurs une large vue sur le fleuve... Mais ce projet laisse irrésolue la question des relations entre les édifices à l'intérieur du quadrilatère.

81. Françoise Fromonot, op. cit., p. 68.

82. Jean Attali, pour la commission «Accueil et vie sociale» écarte Nouvel mais plébiscite Koolhaas, tandis que M. Tonka, rapporteur pour « Architecture - relation avec l'œuvre d'Albert et pertinence du projet » met en avant le schéma de Jean Nouvel et Emmanuel Cattani. Arch. de l'Université Paris VII, boite $\mathrm{n}^{\circ} 58$ - 2. Campus de Jussieu, concours pour la construction de deux bibliothèques universitaires, procès verbal du jury (19 et 24 novembre 1992), daté du 4 février 1993.

83. Le président de Paris VII fait part de sa "grande déception », celui de Paris VI « confirme ces propos». Arch. de l'Université Paris VII, boite $n^{\circ} 58-2$. Campus de Jussieu, concours pour la construction de deux bibliothèques universitaires, procès verbal du jury (19 et 24 novembre 1992), daté du 4 février 1993.

84. Lors des débats, Michel Melot, alors vice-président du conseil supérieur des bibliothèques, écarte le projet de l'O.M.A. "qui n'est pas adapté à une bibliothèque mais à un musée » et "présente de graves problèmes de fonctionnement ». Arch. de l'Université Paris VII, boite $\mathrm{n}^{\circ} 58-$ 2. Campus de Jussieu, concours pour la construction de deux bibliothèques universitaires, procès verbal du jury (19 et 24 novembre 1992), daté du 4 février 1993.

85. Entretien avec Arlette Pailley-Katz et Catherine Tresson, novembre 2006.

86. O.M.A. - Rem Koolhaas et Christophe Corubert, [« Projet pour les bibliothèques de Jussieu »], Campus universitaire de Jussieu, naissance d'une grande bibliothèque, Paris, Sens et Tonka, 1993, 251 p., p. 126-143, p. 126. 
87. Entretien avec Arlette Pailley-Katz et Catherine Tresson, novembre 2006.

88. Arch. de l'Université Paris VII, fonds de la vice-présidence chargée des locaux, boite $n^{\circ} 44$. Projet Erasmus (1993-1995), S.C.A.P., compte rendu de la réunion du 30 juin 1993.

89. Sonya Bertrand, op. cit., http://www.infoscience.fr/travaux/amiante/sommaire.html

90. Le fantasme de la destruction réapparaît toutefois à l'occasion. Ainsi, LeCcanard enchaîné publie des extraits d'un rapport de l'Inspection des finances préconisant la destruction de la tour plutôt que son désamiantage, pour des raisons d'économie. Le Canard enchaîné, 27 avril 2005.

91. La présidence de Paris VII souligne dans les documents d'information sur ce projet que l'extrême imbrication des services à Jussieu est une des causes majeures de son désir d'autonomisation. En 1997 ce projet est déjà bien lancé. Arch. de l'Université Paris VII, document non coté. « Une université dans la ville », daté de décembre 1997.

92. Un troisième établissement scientifique, l'Institut de physique du globe, est présent sur le site. Il est prévu de l'implanter dans l'îlot Cuvier, dont ne seraient conservées que quelques parties anciennes, telles que le pavillon d'entrée et celui des Curie. "Implantation sur l'îlot Cuvier de l'Institut de physique du globe de Paris et de la bibliothèque des sciences de l'univers ", document du Bureau d'information de l'E.P.A. Jussieu, février 2004.

93. Il s'agit du bâtiment Esclangon, élevé à l'emplacement des laboratoires du même nom. Il est l'œuvre d'Alain Sarfati. "Esclangon. Construction d'un nouveau bâtiment sur le campus", document du bureau d'information de l'E.P.A. Jussieu, novembre 2000.

94. D'une surface de $16000 \mathrm{~m}^{2}$, il est destiné à l'accueil des étudiants de premier cycle et est conçu par l'agence Périphériques. "Un bâtiment pédagogique de $16000 \mathrm{~m}^{2}$ sur le campus ", document du bureau d'information de l'E.P.A. Jussieu, juin 2003.

95. «La rénovation du secteur ouest du campus de Jussieu », document du bureau d'information de l'E.P.A. Jussieu, février 2005.

\section{RÉSUMÉS}

Pris entre les critiques émanant des défenseurs du patrimoine parisien et les plaidoyers favorables des historiens de l'architecture contemporaine, le campus de Jussieu, élevé à partir de 1964 sur le site de l'ancienne Halle aux vins, reste un objet architectural difficile à appréhender, d'autant plus que le scandale de santé publique de l'amiante lui confère depuis dix ans une triste réputation. On émet ici l'hypothèse que «l'inachèvement de Jussieu ", constaté après 1972, ne se limite pas à l'interruption du programme architectural tel qu'il avait été défini par Édouard Albert dix ans plus tôt : il s'agit également des difficultés nées de la difficile cohabitation entre deux universités qui s'opposent sur bien des points, alors que le projet de départ était prévu pour accueillir une structure unifiée et très hiérarchisée. Il s'agit enfin de l'incompréhension jamais dépassée entre architectes et usagers de ce lieu. On ne peut prendre pleinement la mesure de ce triple échec qu'en rappelant l'ambition démesurée de ce projet de quelque $400000 \mathrm{~m}^{2}$, tant dans le domaine scientifique que sur le plan institutionnel, aussi bien en termes d'architecture publique que de programmation artistique. Dès lors, les solutions proposées depuis plus de trente ans pour l'aménagement du site sont envisagées non seulement comme autant de projets architecturaux visant à remédier au caractère foncièrement inesthétique d'un bâtiment inachevé, mais aussi, et surtout, comme une série de solutions imaginées pour rendre possible une véritable vie universitaire sur le campus, ambition qui va bien au-delà de l'accroissement de la surface bâtie disponible. La crise de l'amiante, après 1996, marque le début de la rénovation du 
site, en même temps qu'est choisie une solution radicale : la dissociation des deux universités auparavant étroitement imbriquées et le départ de l'une d'entre elles vers un autre lieu. Bâtiment exceptionnel par son histoire, le campus de Jussieu est également emblématique des difficultés récurrentes de l'architecture universitaire dans notre pays. On tentera en conclusion d'en livrer une explication.

Torn between the criticism from the defenders of Parisian heritage and the favourable pleas of architectural historians, the Jussieu campus, erected from 1964 on the site of the old wine market, is still difficult to apprehend as an architectural object, all the more so since the abestos health scandal brought it into disrepute ten years ago. We suggest the possibility that the "incompletion of Jussieu", notified after 1972, does not only owe to the fact that the architectural program Edouard Albert defined ten years ago has not been fully executed : it's also about some matters arisen from the problematic cohabitation of two universities that conflict with each other on many points, whereas the initial project was to have housed one unified and wellorganized structure. At last it is about the architects and the users always failing to understand each other. These three failures can be fully sized up only by remembering the immoderate ambition of this approximately 400.000 square meters project, scientifically or institutionally speaking, whether speaking of public architecture or artistic planning. That being the case, the planning solutions put forward for more than thirty years are not only designed as architectural projects meant to put right the unsightly character of an unachieved building, but also and above all as a series of solutions conceived of so as to make a true university life possible on the campus - ambition that goes far beyond the enlargement of the available surface area. The abestos crisis of 1996 marks the beginning of the renovation program of the facilities, while a complete break with past practices is decided : the dissociation of the two universities formerly tightly interlinked and the departure of one of them to another facility. As an exceptional building regarding its history, the Jussieu campus is also emblematic of the recurrent difficulties of university architecture in our country. As a conclusion we will try to give an explanation.

Der ab 1964 auf dem Gebiet der alten Wein-Markthalle gebaute Campus von Jussieu ist als architektonisches Objekt zwischen der heftigen Kritik der Verteidiger des Pariser Denkmalschutzes und dem positiven Urteil der zeitgenössischen Architekturhistoriker schwierig zu bewerten, zumal er aufgrund des öffentlichen Gesundheitsskandals wegen der Asbestverseuchung seit zehn Jahren einen besonders schlechten Ruf genießt. Den nach 1972 anerkannten Charakter der «Unvollendung Jussieus » erklärt sich laut dem Autor des Artikels nicht einfach durch die damalige Unterbrechung des schon zehn Jahre früher von Edouard Albert festgelegten Bauprogramms. Es liegt ebenso an der problematischen Verquickung zweier in mancher Hinsicht entgegengesetzter Universitäten auf einem Areal, das ursprünglich für eine einzige sehr hierarchisch angelegte Einheit geplant wurde. Es zeugt ebenfalls von der nie beseitigten Diskrepanz zwischen den Absichten der Architekten und den Erwartungen der Anlagenutzer. Dieses enorme dreifache Scheitern des Unternehmens entspringt aus dem ehrgeizigen Gigantismus des Projekts $(400.000 \mathrm{~m} 2)$ in wissenschaftlichen und institutionellen Bereichen, so wie die öffentliche Architektur und das künstlerische Programm betreffend. Die seit über dreißig Jahre laufenden etlichen architektonischen Projekte an Jussieu, die sich zwar auf das vollkommen hässliche Aussehen eines unvollendeten Gebäudes beziehen, versuchen aber vor allem, ein echtes Universitätsleben auf dem Campus zu ermöglichen und nehmen sich dabei viel mehr vor als nur die Erweiterung der beständigen Baufläche. Mit der Asbestkrise fing ab 1996 das Renovierungsprogramm der Fakultät an. Gleichzeitig wurde eine radikale Maßnahme getroffen: die Trennung der zwei Universitäten, die vorher eng verbunden waren, und die Verlagerung einer von beiden auf ein anderes Areal. Das von seiner Geschichte her auß ergewöhnliche Gebäude des Campus von Jussieu ist repräsentativ für die sich wiederholenden 
Schwierigkeiten Frankreichs mit den universitären Bauten. Zum Schluss wagt der Autor eine Erläuterung zu diesem Phänomen.

\section{AUTEUR}

\section{CHRISTIAN HOTTIN}

Christian Hottin, archiviste paléographe (1997) et conservateur du patrimoine dans la spécialité archives (2001), a travaillé de 2001 à 2004 comme conservateur aux Archives nationales (centre des archives du monde du travail), où il est était en charge des fonds d'architectes, d'ingénieurs et d'urbanistes. Il est actuellement chef de la mission ethnologie à la direction de l'architecture et $\mathrm{du}$ patrimoine et à ce titre représentant du ministère de la culture au sein du LAHIC (équipe du IIAC). Il rédige une thèse de doctorat (Éphé, IV ${ }^{\mathrm{e}}$ section) consacrée à l'architecture de l'enseignement supérieur à Paris au XIX ${ }^{\mathrm{e}}$ et $\mathrm{XX}^{\mathrm{e}}$ siècles. Sa thèse de l'École des chartes portant sur les décors universitaires a été publiée en 2001 (Quand la Sorbonne était peinte, Paris, Maisonneuve et Larose, 2001, 312 p.) ; il est en outre l'auteur de divers travaux sur l'architecture et les représentations du monde de l'université, des archives ou des bibliothèques, réalisés dans le cadre d'expositions ou d'ouvrages collectifs, et consultables en ligne sur le serveur HAL-SHS (URL personnelle : http://hal.archives-ouvertes.fr/aut/hottin/). Il publie en 2007 le catalogue du patrimoine peint et sculpté de la Sorbonne. Adresse électronique : christian.hottin@wanadoo.fr 